\title{
Effects of the eccentricity of the primaries in powered Swing-By maneuvers
}

\author{
Alessandra F.S. Ferreira ${ }^{a, *}$, Antônio F.B.A. Prado ${ }^{a}$, Othon C. Winter ${ }^{b}$, \\ Denilson P.S. Santos ${ }^{\mathrm{c}}$ \\ a Instituto Nacional de Pesquisas Espaciais - INPE, 12227-010 São José dos Campos, SP, Brazil \\ ${ }^{\mathrm{b}}$ Grupo de Dinâmica Orbital e Planetologia, Univ. Estadual Paulista-UNESP, 12516-410 Guaratinguetá, SP, Brazil \\ ${ }^{\mathrm{c}}$ Univ. Estadual Paulista - UNESP, 13876-750 São João da Boa Vista, SP, Brazil
}

Received 18 July 2016; received in revised form 19 January 2017; accepted 23 January 2017

Available online 1 February 2017

\begin{abstract}
The present paper studies the powered Swing-By maneuver when performed in an elliptical system of primaries. It means that there is a spacecraft travelling in a system governed by the gravity fields of two bodies that are in elliptical orbits around their center of mass. The paper particularly analyzes the effects of the parameters relative to the Swing-By $\left(V_{\text {inf-- }}, r_{p}, \psi\right)$, the orbit of the secondary body around the primary one $(e, v)$ and the elements that specify the impulse applied $(\delta V, \alpha)$ to the spacecraft. The impulse is applied when the spacecraft passes by the periapsis of its orbit around the body, where it performs the Swing-By, with different magnitudes and directions. The inclusion of the orbital eccentricity of the primaries in this problem makes it closer to reality, considering that there are many known systems with eccentricities different from zero. In particular, there are several moons in the Solar System which orbits are not circular, as well as some smaller bodies, like the dwarf planet Haumea and its moons, which have eccentricities of 0.25 or even larger. The behavior of the energy variation of the spacecraft is shown in details, as well as the cases where captures and collisions occur. The results show the conditions that optimize this maneuver, according to some given parameters, and how much can be obtained in terms of gains or losses of energy using the best conditions found by the algorithm developed here.
\end{abstract}

(C) 2017 COSPAR. Published by Elsevier Ltd. All rights reserved.

Keywords: Astrodynamics; Powered Swing-By maneuvers; Spacecraft trajectories; Impulsive maneuvers; ERTBP

\section{Introduction}

The "Powered Swing-By Maneuver" is a technique where a spacecraft approaches a celestial body and uses its gravity field to modify its trajectory, but combined with an impulse delivered to the spacecraft by a propulsion system. It is a more complex maneuver compared to the pure gravity "Swing-By Maneuver", where only the gravity field

\footnotetext{
* Corresponding author.

E-mail addresses: aleferrazsilva@hotmail.com (A.F.S. Ferreira), antonio.prado@inpe.br (A.F.B.A. Prado), ocwinter@gmail.com (O.C. Winter), denilson.paulo@gmail.com (D.P.S. Santos).
}

of the celestial body performs the modification of the trajectory. The advantage of the "Powered Swing-By Maneuver" is to have more energy and more flexibility in the effects, because the application of the impulse during the close approach gives larger variations of energy when compared to a maneuver that uses the impulse far from the close approach point (Prado, 1996).

The pure gravity maneuver is well studied in the literature, and many practical applications are known. Minovitch (1961) authored one of the first and most important documents in this field. He showed the basic facts about Swing-Bys when applied to spacecraft trajectories. A description of the works developed by Minovitch is 
available in Dowling et al. (1991, 1992). Regarding practical applications, Flandro (1966) designed the Voyager missions, based in the equations developed by Minovitch. After that, Swing-Bys in the large planets of the Solar System were used to get energy to help the spacecrafts to reach their goals. The Galileo mission also used the pure gravity Swing-By maneuver, like shown in D'Amario et al. (1981, 1982) and Byrnes and D'Amario (1982). Other missions using this maneuver are shown in NASA $(2010,1999$ 2012). The missions Messenger and BepiColombo to Mercury are also very important and they are described in McNutt et al. (2004, 2006), Grard (2006) and Jehn et al. (2008). Another interesting aspect of this problem is the possibility of using multiple swing-bys, as shown in Ross and Scheeres (2007) and Formiga and Prado (2014).

This maneuver can also contribute to the capture of spacecrafts by celestial bodies, like shown in Nock and Upholf (1979), Lynam et al. (2011) and Brasil et al. (2015). Some authors considered this maneuver combined with a passage of the spacecraft by the atmosphere of a planet, known as "aero-gravity assisted maneuver", as shown in Lewis and McRonald (1992), Sims et al. (1995, 2000), Lohar et al. (1996), Bonfiglio et al. (2000), Lavagna et al. (2005), Armellin et al. (2007) and Gomes et al. (2015). A combination of low thrust and gravity assists are used in McConaghy et al. (2003), Okutsu et al. (2006) and Santos et al. (2008). Maneuvers considering a cloud of particles are studied in Gomes and Prado (2010). The particular case of powered Swing-By maneuvers, in the circular case, are studied in Prado (1996), Silva et al. (2013a, 2013b) and Ferreira et al. (2015).

The present paper studies a version of this problem where the powered Swing-By maneuver is made in a situation where the two primaries of the system are in elliptical orbits around their common center of mass. Elliptical systems are numerous in the real world and there are several studies related to this dynamics. Broucke (1969) presented a study about the stability of periodic orbits in the restricted elliptical three-body problem. A good example of a highly eccentric system in the Solar System is the dwarf planet Haumea, which has a moon with eccentricity close to 0.25 (Sanchez et al., 2016). A first look at maneuvers in this system is shown in Ferreira et al. (2016). Also very related to the present research, Prado (1997) studied the pure gravity SwingBy maneuver (without impulse) in the elliptical case.

In this sense, this research complements those previous papers by studying the powered version of the maneuver, but performed in systems where the orbits of the primaries are elliptical.

The mathematical model given by the "Elliptical Restricted Three-Body Problem (ERTBP)" is well known in the literature (Szebehely, 1967) and it is used here. It focuses on the study of the motion of a body with negligible mass around two massive bodies that are orbiting their common center of mass in elliptical orbits. There are no analytical solutions and numerical integration is the most common approach used to search for trajectories under this model. This is the technique used in the present paper.
The main goal of the present paper is to analyze the evolution of the energy of the spacecraft as a function of the parameters that describe the maneuver. Concerning the gravity part of the maneuver, the parameters are: $V_{\text {inf-- }}$, the velocity of the spacecraft when approaching the celestial body; $r_{p}$, the radius of the periapsis, which is the distance of the closest approach between the spacecraft and the celestial body during the whole maneuver; and $\psi$, the angle of approach, which is the angle between the line of periapsis and the line connecting both primaries. Regarding the impulse, the parameters required to specify the maneuver are: $\delta V$, the magnitude of the impulse; and $\alpha$, the angle that defines the direction of the application of the impulse, measured with respect to the direction of the motion of the spacecraft. It is also necessary to specify the orbits of the primaries, which is made by given the orbital eccentricity $(e)$ and the true anomaly of the secondary body in its orbit around the primary at the moment where the maneuver is performed $(v)$. The semi-major axis of the orbit is not specified, because the canonical system of units is used and so this parameter is one.

To study this problem, an algorithm is developed to numerically integrate the equations of motion under different initial conditions to find the trajectories of interest. This algorithm makes the numerical integrations of the orbit of the spacecraft in positive times (with the inclusion of the impulse) and negative times (without the inclusion of the impulse). Those numerical integrations are performed until the spacecraft is far from the secondary body, so they allow the calculations of the energy before and after the maneuver, which gives the total variations. Using this technique, the results focus on measuring the variation of energy given by the Powered Swing-By Maneuver, to find the best direction to apply the impulse to reach some specific goals, like maximizing or minimizing the energy gains or losses.

\section{Dynamical system}

As explained before, the studies performed here are based on the mathematical model given by the "Elliptical Restricted Three-Body Problem (ERTBP)“" (Szebehely, 1967). The system is formed by two bodies with finite masses, called primaries and denoted by $M_{1}$ and $M_{2}$; being $M_{1}$ the main body with the largest mass and $M_{2}$ the secondary body. There is also a third body with negligible mass, called $M_{3}$, that moves under the gravitational forces of the two primaries. In the present case, it represents a spacecraft moving around the two bodies. This spacecraft makes a close approach with $M_{2}$, whose study is the main goal of the present paper. The study is limited to the plane of the primaries, since this is the case with more practical applications. The two primaries $M_{1}$ and $M_{2}$ are moving around their common center of mass in elliptical orbits. The canonical system of units is used and it is abbreviated by "c.u.". It has the following properties: the mass of $M_{2}$ is given by $\mu=\frac{m_{2}}{\left(m_{1}+m_{2}\right)}$ (with $m_{1}$ and $m_{2}$ the real masses of $M_{1}$ and $\left.M_{2}\right)$ and the mass of $M_{1}$ is $(1-\mu)$, such that the total 
mass of the system is unitary; the gravitational constant is also unitary; the unit of distances is the semi-major axis of the orbit of the two primaries and the unit of time is defined such that the orbital period of the primaries is $2 \pi$.

To write the equations of motion, the fixed reference system (Szebehely, 1967) is used in the study presented here. There are other choices, like the rotating and the rotatingpulsating reference systems (Szebehely, 1967). They are equivalent, and the choice for the fixed system is made just to simplify the transformations of variables. In this system, the equations of motion are given by Eq. (1).

$\ddot{x}=\frac{-(1-\mu)\left(x-x_{1}\right)}{r_{1}^{3}}-\frac{\mu\left(x-x_{2}\right)}{r_{2}^{3}}$,

$\ddot{y}=\frac{-(1-\mu)\left(y-y_{1}\right)}{r_{1}^{3}}-\frac{\mu\left(y-y_{2}\right)}{r_{2}^{3}}$

where $r_{1}$ is the distance between $M_{1}$ and $M_{3} ; r_{2}$ the distance between $\quad M_{2}$ and $M_{3} ; \quad x_{1}=-\mu r \cos v, \quad y_{1}=-\mu r \sin v$, $x_{2}=(1-\mu) r \cos v$ and $y_{2}=(1-\mu) r \sin v$ are the positions of $M_{1}$ and $M_{2}$, respectively, in the fixed reference system originating in the center of mass of the system.

The distance between the primaries is given by $r=\frac{a\left(1-e^{2}\right)}{1+e \cos v}$, where $a$ is the semi-major axis $(a=1$ as explained before) and $v$ is the true anomaly of $M_{2}$ relative to $M_{1}$. Fig. 1 describes the maneuver and show the important variables involved.

The algorithm developed to study this problem integrates the equations of motion, so it is possible to obtain the energy variation of the spacecraft, calculated by the difference of the energy after and before the maneuver. The final orbit is the one obtained after the Powered SwingBy maneuver. The magnitude and direction of the impulse is varied to search for the best values, according to the goal of the maneuver. Therefore, the final algorithm has the steps shown next.

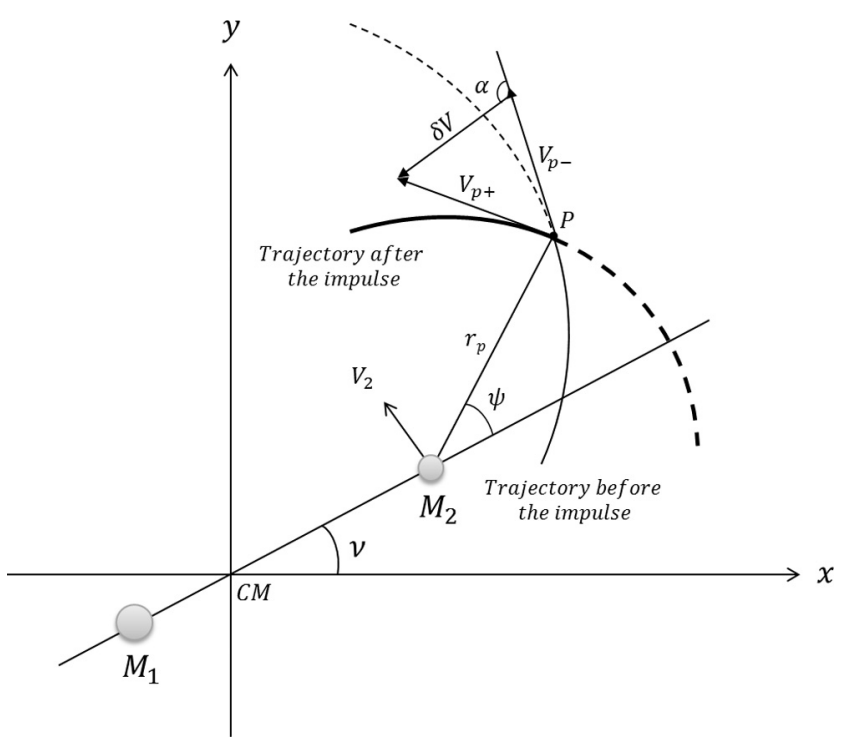

Fig. 1. Description of the maneuver. (i) The initial conditions of the spacecraft are set at the periapsis of the orbit of the spacecraft around $\mathrm{M}_{2}$ $\left(x_{p}, y_{p}, \dot{x}_{p}\right.$ and $\left.\dot{y}_{p}\right)$. This is done by using the initial values of the variables described before: $V_{\text {inf- }}$, the velocity of approach of the spacecraft with respect to $M_{2}$; $r_{p}$, the periapsis distance; $\psi$, the angle of approach; $e$, the orbital eccentricity of the primaries; and $v$, the true anomaly of the secondary body at the moment where the maneuver is performed;

$$
\left\{\begin{aligned}
x_{p} & =\frac{(1-\mu)\left(1-e^{2}\right)}{1+e \cos v} \cos v+r_{p} \cos (\psi+v) \\
y_{p} & =\frac{(1-\mu)\left(1-e^{2}\right)}{1+e \cos v} \sin v+r_{p} \sin (\psi+v) \\
\dot{x}_{p} & =\frac{(1-\mu) e \sin v}{\sqrt{1-e^{2}}} \cos v-\frac{(1-\mu)(1+e \cos v)}{\sqrt{1-e^{2}}} \sin v \\
& -\sqrt{V_{i n f-}^{2}+\frac{2 \mu}{r_{p}}} \sin (\psi+v) \\
\dot{y}_{p} & =\frac{(1-\mu) e \sin v}{\sqrt{1-e^{2}}} \sin v+\frac{(1-\mu)(1+e \cos v)}{\sqrt{1-e^{2}}} \cos v \\
& +\sqrt{V_{i n f-}^{2}+\frac{2 \mu}{r_{p}}} \cos (\psi+v)
\end{aligned}\right.
$$

(ii) The orbit is integrated backwards in time until the spacecraft reaches a distance where it can be assumed that the motion spacecraft-main primary is keplerian. This value is in the range from 0.3 to 0.5 c.u. ( 1 canonical unit $=1$ c.u. $=M_{1}-M_{2}$ distance). A test is made to choose the best value. At this point all the important parameters of the spacecraft before the maneuver can be measured: velocity, position, energy, angular momentum, etc.

(iii) After that, the initial conditions of the spacecraft are placed again at the periapsis, like done in step (i). The impulse is applied, using the variables: $\delta V$, the magnitude of the impulse; and $\alpha$, the angle formed by the impulse vector and the direction of the motion of the spacecraft. $\beta$ is the angle between $\vec{V}_{p-}$ and $\dot{x}$ axis. After that the orbit is integrated forward in time, until the spacecraft reaches again a distance where it can be assumed that the motion spacecraft-main primary is keplerian, in the same form made in step (i). At this point all the important parameters of the spacecraft after the maneuver are measured: velocity, position, energy, angular momentum, etc.

$$
\left\{\begin{array}{c}
x_{p}=\frac{(1-\mu)\left(1-e^{2}\right)}{1+e \cos v} \cos v+r_{p} \cos (\psi+v) \\
y_{p}=\frac{(1-\mu)\left(1-e^{2}\right)}{1+e \cos v} \sin v+r_{p} \sin (\psi+v) \\
\dot{x}_{p}=\frac{(1-\mu) e \sin v}{\sqrt{1-e^{2}}} \cos v-\frac{(1-\mu)(1+e \cos v)}{\sqrt{1-e^{2}}} \sin v \\
-\sqrt{V_{i n f-}^{2}+\frac{2 \mu}{r_{p}}} \sin (\psi+v)+\frac{\delta V \cos (\beta-\alpha)}{V_{2}} \\
\dot{y}_{p}=\frac{(1-\mu) e \sin v}{\sqrt{1-e^{2}}} \sin v+\frac{(1-\mu)(1+e \cos v)}{\sqrt{1-e^{2}}} \cos v \\
+\sqrt{V_{i n f-}^{2}+\frac{2 \mu}{r_{p}}} \cos (\psi+v)+\frac{\delta V \sin (\beta-\alpha)}{V_{2}}
\end{array}\right.
$$

(iv) Based on the parameters before and after the maneuver, the variations made by the maneuver can be 
obtained just by direct subtractions of the quantities involved. Then, it is possible to map the variations of the desired quantities as a function of all the variables, to search for the best direction to apply the impulse for a given geometry and magnitude of the impulse.

Fig. 1 shows a description of the maneuver. The $x$ and $y$ axes define the fixed reference system originating in the center of mass of the system $(C M) . V_{2}$ is the velocity of $M_{2}$ with respect to $M_{1} ; v$ is the true anomaly of the secondary body, describing the position of $M_{2}$ with respect to $M_{1}$. $\psi$ (angle of approach) and $r_{p}$ (periapsis distance) specify the initial position of the spacecraft, defining its orbit. $P$ is the periapsis of the orbit and the point where the impulse is applied. The black thin solid line represents the trajectory of the spacecraft before the impulse, which ends at the periapsis, where the impulse is applied. The dashed thin line is an imaginary continuation of this orbit, which would be the orbit of the spacecraft if the impulse was not applied. At the point $P$ the second part of the orbit starts (black bold line), representing the trajectory of the spacecraft after the impulse. The dashed black bold line represents the trajectory that a spacecraft would have before the impulse, if its orbit was the one after the impulse. The angle $\alpha$ describes the direction of the impulse, measured with respect to the direction of motion of the spacecraft. If $-180^{\circ}<\alpha<0^{\circ}$, the impulse has a component in the direction of $M_{2}$, making the spacecraft to get closer to it. If $0^{\circ}<\alpha<180^{\circ}$, the impulse has a component in the opposite direction of $M_{2}$, making the spacecraft to move away from it. For $\alpha<-90^{\circ}$ or $\alpha>90^{\circ}$ there is a component of the impulse that is opposite to the direction of the motion of the spacecraft, making it to decelerate. In the interval $-90^{\circ}<\alpha<90^{\circ}$ the spacecraft is accelerated by the impulsive maneuver, because the impulse is applied in the direction of the motion of the spacecraft. The magnitude of the impulse is denoted by $\delta V . V_{P-}$ is the velocity of the spacecraft at periapsis before the impulse and $V_{P+}$ is the velocity of the spacecraft after the impulse.

\section{Results}

The results consist in plotting maps showing the energy variation as a function of the angle that defines the direction of the impulse ( $\Delta E$ vs. $\alpha$ ) for different initial conditions. A system with the mass parameter $\mu=0.01214$, sizes and distances equivalent to the Earth-Moon system was used in all the simulations, but different values for the eccentricity were tested to measure the influence of this parameter: $e=0.1, e=0.3$ and $e=0.5$. The periapsis radius used in the simulations was $r_{p}=1.1$ radius of the secondary body, to get large effects from the gravity part of the maneuver. For the angle of approach $\psi$, several values were used, to cover the full range of possibilities, from $0^{\circ}$ to $360^{\circ}$, in steps of $45^{\circ}$. The true anomaly $(v)$ received the values: $0^{\circ}, 90^{\circ}, 180^{\circ}$ and $270^{\circ}$, so covering the regions of maximum gains, maximum losses and zero variation of energy, with respect to the pure gravity maneuver. The magnitude of the impulse $\delta V$ ranges from $0.1 \mathrm{~km} / \mathrm{s}$ to $4.0 \mathrm{~km} / \mathrm{s}$. It gives a total of 960 different sets of initial conditions. They were numerically integrated with the direction of the impulse $\alpha$ ranging from $-180^{\circ}$ to $180^{\circ}$, in steps of $1.0^{\circ}$, thus resulting in 346,560 trajectories with different energy variations.

\subsection{Effects of the geometry of the maneuver}

Fig. 2 and Table 1 show the results when using eccentricity equal to 0.1 , true anomaly equal to $0^{\circ}$ (so $M_{2}$ is at the periapsis of its orbit around $M_{l}$ when the close approach occurs), and different values for the angle of approach $\psi$ and the magnitude of the impulse. There is one curve for each value of the magnitude of the impulse and one plot for each value of the angle of approach. The true anomaly zero was chosen because this is the location that gives the maximum variation of energy for the pure gravity assisted maneuver, if all the other parameters are fixed (Prado, 1997). Due to the similarity of the results, only the plots for $\psi=0^{\circ}, 90^{\circ}, 180^{\circ}$ and $270^{\circ}$ are shown. Several characteristics are visible in the results. The first one is the expected increase of the energy variation with the magnitude of the impulse. Although this result is expected, the plots shown here can quantify those increases, which help to decide the best magnitude to be used in a real maneuver. The end points of the lines shown in Fig. 2 are due to regions of captures or collisions.

Table 1 shows better some important points. To understand many of those points it is necessary to take into account that the impulse modifies the trajectory of the spacecraft in several aspects. It includes the modification of the angle of approach. So, the values for the angle of approach shown in the results are the ones with respect to a pure gravity maneuver. This value is used because it is necessary to design the orbit that takes the spacecraft to the periapsis of the orbit to receive the impulse but, after the impulse is applied, the real values of the angle of approach is modified and it depends on the application of the impulse.

For $\psi=0^{\circ}$, where there is no energy variation for a pure gravity maneuver, the optimum direction of the impulse is near zero, which means that it is applied in the direction of the motion of the spacecraft. It is consistent with the idea that, since there is no contribution in the pure gravity maneuver, it is important to get the most variation of energy possible from the impulse. This situation occurs when applying the impulse in the direction of the motion of the spacecraft. The direction of the impulse is near zero, but always positive, to send the spacecraft away from $\mathrm{M}_{2}$, which makes the real angle of approach to be in the region of gains of energy $\left(180^{\circ}<\psi<360^{\circ}\right)$. This value is reduced when the magnitude of the impulse increases, because in this situation the powered part of the maneuver has a 


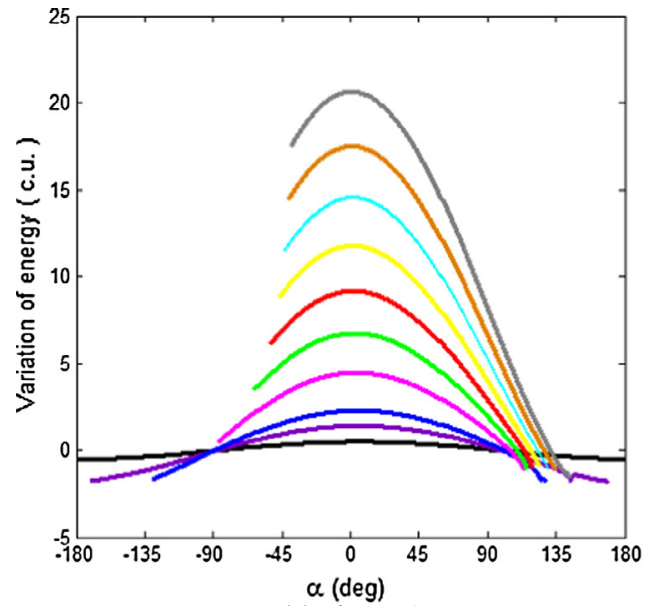

(a) $\psi=0^{\circ}$

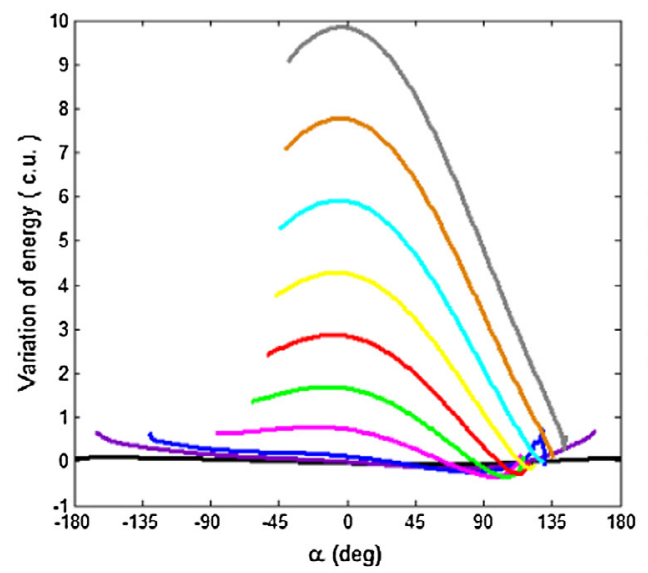

(c) $\psi=180^{\circ}$

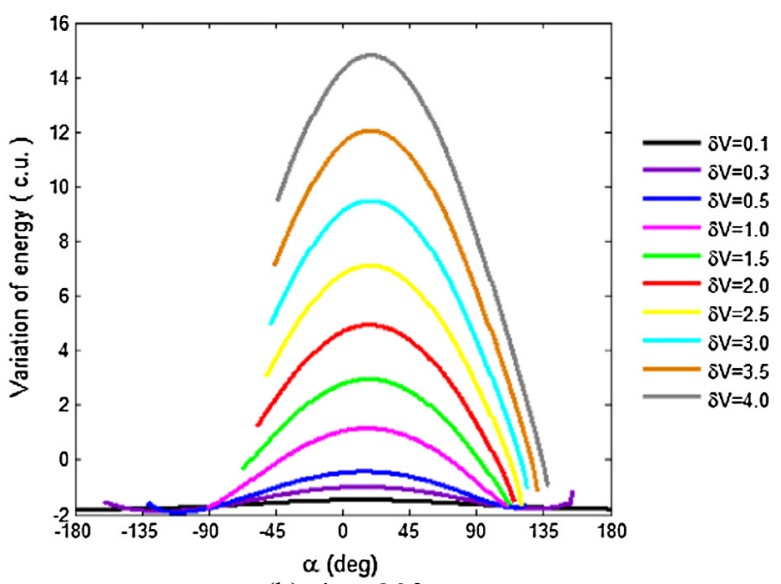

(b) $\psi=90^{\circ}$

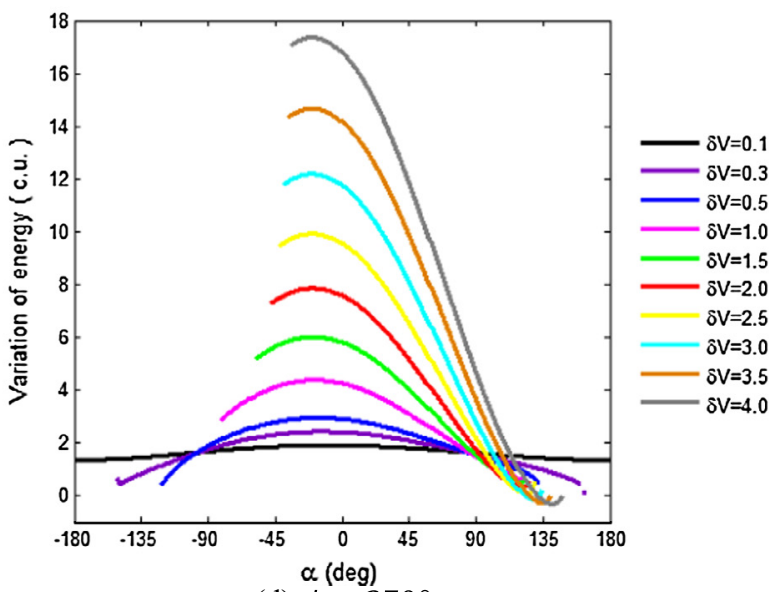

(d) $\psi=270^{\circ}$

Fig. 2. Variation of energy as a function of the direction of the impulse for $e=0.1$ and $v=0^{\circ}$.

Table 1

Maximum energy variation and the corresponding $\alpha$ for the case of Fig. $2\left(e=0.1\right.$ and $\left.v=0^{\circ}\right)$.

\begin{tabular}{|c|c|c|c|c|c|c|c|c|}
\hline \multirow[t]{2}{*}{$\delta V(\mathrm{~km} / \mathrm{s})$} & \multicolumn{2}{|l|}{$\psi=0^{\circ}$} & \multicolumn{2}{|l|}{$\psi=90^{\circ}$} & \multicolumn{2}{|l|}{$\psi=180^{\circ}$} & \multicolumn{2}{|l|}{$\psi=270^{\circ}$} \\
\hline & $\Delta E_{\max }$ (c.u.) & $\alpha\left(^{\circ}\right)$ & $\Delta E_{\max }$ (c.u.) & $\alpha\left(^{\circ}\right)$ & $\Delta E_{\max }$ (c.u.) & $\alpha\left(^{\circ}\right)$ & $\Delta E_{\max }$ (c.u.) & $\alpha\left({ }^{\circ}\right)$ \\
\hline 0.1 & 0.4841 & 6.0 & -1.4616 & 13.0 & 0.0941 & -154.0 & 1.9248 & -9.0 \\
\hline 0.3 & 1.3987 & 6.0 & -0.9778 & 14.0 & 0.6733 & 165.0 & 2.4384 & -13.0 \\
\hline 0.5 & 2.2784 & 6.0 & -0.4206 & 15.0 & 0.7087 & 129.0 & 2.9611 & -16.0 \\
\hline 1.0 & 4.462 & 4.0 & 1.1637 & 17.0 & 0.7816 & -21.0 & 4.3881 & -18.0 \\
\hline 1.5 & 6.734 & 3.0 & 2.9572 & 18.0 & 1.6928 & -13.0 & 6.0203 & -19.0 \\
\hline 2.0 & 9.1525 & 3.0 & 4.9422 & 18.0 & 2.8683 & -9.0 & 7.8651 & -19.0 \\
\hline 2.5 & 11.7415 & 2.0 & 7.1177 & 19.0 & 4.2817 & -7.0 & 9.9222 & -20.0 \\
\hline 3.0 & 14.5125 & 2.0 & 9.4857 & 19.0 & 5.9199 & -5.0 & 12.1901 & -20.0 \\
\hline 3.5 & 17.4721 & 1.0 & 12.0479 & 19.0 & 7.7757 & -4.0 & 14.6673 & -20.0 \\
\hline 4.0 & 20.625 & 1.0 & 14.8058 & 19.0 & 9.8448 & -4.0 & 17.3524 & -20.0 \\
\hline
\end{tabular}

larger participation, compared to the gravity part of the maneuver.

Considering the case $\psi=90^{\circ}$, where there are maximum losses of energy in the pure gravity maneuver, the optimum directions for the impulse increases, but remains in the positive region, also giving a component for the velocity of the spacecraft opposite to the direction of $M_{2}$. It is in agreement with the principle that, since there are losses of energy from the gravity part of the maneuver, it is better to place the spacecraft as far as possible from the celestial body, such that it minimizes the loss of energy. The impulse also modifies the angle of approach, removing it from the value of the maximum loss of energy $\left(\psi=90^{\circ}\right)$. On the other side, to get the most variation of energy possible from the impulse, its direction should be as close as possible to the direction of the motion of the spacecraft. In order to 
have a compromise between those two effects, the optimum solutions have positive values for the direction of the impulse. The angle representing the direction of the impulse gets larger when the magnitude of the impulse increases, representing the fact that an increase in the magnitude allows stronger modifications in the orbit, making large changes in the angle of approach. Since this situation represents the maximum loss of energy, modifications in this geometry give effects much stronger than in the previous case, $\psi=0^{\circ}$, which is near a region of no effects from the gravity part of the maneuver.

The case $\psi=180^{\circ}$ is another situation where there is no energy variation in the pure gravity maneuver. The main difference from the case $\psi=0^{\circ}$ is that, now, a retrograde impulse increases the turning angle of the spacecraft and shift the angle of approach to the region of energy gains $\left(180^{\circ}<\psi<360^{\circ}\right)$. It is visible for impulses up to $0.5 \mathrm{~km} /$ s. After this value the impulse is applied in the direction of the motion of the spacecraft, to get more energy from the powered part of the maneuver, but with negative values, to make the spacecraft to get closer to $M_{2}$ to increase the gains coming from the gravity part of the maneuver, since now it gives gains of energy. Remember that a negative value also shift the angle of approach to the region of gains of energy.

The geometry with $\psi=270^{\circ}$ represents the case with maximum gains of energy for the pure gravity maneuver. So, it is better to make the spacecraft to get as close as possible to the celestial body. The optimum directions for the impulse moves to the negative region, to send the spacecraft to the direction of $M_{2}$. When the magnitude of the impulse increases, the direction of the impulse increases in magnitude. It happens because larger impulses can make stronger effects in the geometry, which is once again in a region very sensitive to this effect, since it is near the region of maximum gains of energy.

In general, it is noted that, for small values of the magnitude of the impulse, the gravity gains of the maneuver dominates the scenario and the maximum and minimum gains of energy are located in the same regions predicted by the standard maneuver, $\psi=270^{\circ}$ and $\psi=90^{\circ}$, respectively. In the cases of zero gain of energy for the pure gravity maneuver $\left(\psi=0^{\circ}\right.$ and $\left.\psi=180^{\circ}\right)$, the impulse is applied such that it shifts the angle of approach to the region of gains of energy. Another observation that can be made is related to the sign of the variation of energy. It is possible to find regions of positive variations of energy even in geometries of maximum losses of energy $\left(\psi=90^{\circ}\right)$. In the situations of neutral variation of energy for the pure gravity maneuver $\left(\psi=0^{\circ}\right.$ and $\left.\psi=180^{\circ}\right)$, the powered maneuver can always obtain gains of energy, as expected. The case $\psi=0^{\circ}$ gives larger variations of energy compared to the case $\psi=180^{\circ}$. The reason in that to shift the angle of approach to the region of gains of energy, in the case $\psi=0^{\circ}$, the impulse has to be applied in the direction of motion of the spacecraft, so getting more effects from the powered part of the maneuver. It is the opposite in the case $\psi=180^{\circ}$, where the impulse needs to be applied in the opposite direction of motion of the spacecraft, to get more energy from the gravity part of the maneuver, but then it losses energy from the powered part of the maneuver.

Fig. 3 and Table 2 show the effects of the true anomaly of the secondary body in the variation of energy, as a function of the same parameters used in Fig. 2. The eccentricity used is $e=0.1$ and the true anomaly assumes the values: $0^{\circ}, 90^{\circ}, 180^{\circ}$, and $270^{\circ}$. The results show that the optimum directions to apply the impulse are always negative, since the angle of approach is $270^{\circ}$ and the maneuver tries to get the largest possible variation of energy from the gravity part of the maneuver. The true anomaly does not change this fact. To understand the effects of this parameter, it is necessary to remember that the variation of energy is proportional to the velocity of $M_{2}$, which is higher at the periapsis. This rule is followed by the first line of Table 2, for a magnitude of the impulse of $0.1 \mathrm{~km} / \mathrm{s}$. The variation of energy is maximum for $v=0^{\circ}$ and minimum for $v=180^{\circ}$. For larger values of the magnitude of the impulse, it is very difficult to make predictions for the energy variations. The important parameter is the angle between the velocity vector of $M_{2}$ with respect to $M_{1}$ and the velocity of the spacecraft with respect to $M_{2}$ at periapsis. Those variables are modified by the true anomaly of $M_{2}$ and the impulse. The final result of these effects is an inversion in the variation of energy and the maximum gains of energy moves to the true anomaly $180^{\circ}$, with the minimum at $v=0^{\circ}$. This is done to maximize the angle between the velocity vector of $M_{2}$ with respect to $M_{1}$ and the velocity of the spacecraft at periapsis. It means that the results shown here are required to find the best maneuvers.

Fig. 4 and Table 3 are made to measure the effects of the eccentricity in this maneuver. The eccentricity is now 0.3 , the angle of approach is $270^{\circ}$ and the true anomaly is varied to assume the same values used before: $0^{\circ}, 90^{\circ}, 180^{\circ}$ and $270^{\circ}$. Once again, all the directions of the impulses are negative, sending the spacecraft to the direction of $M_{2}$ to get more variation of energy from the gravity part of the maneuver. The magnitude of this angle increases with the magnitude of the impulse, because larger impulses can make stronger effects in the geometry, leading to larger gains in energy from the combined maneuver. It is also noted that the maximum gains of energy moves from the angle of approach $270^{\circ}$ when the impulse increases, representing the fact that the geometry is modified by the impulse and the true anomaly of $M_{2}$. The increase of the eccentricity modifies the point where this inversion occurs and now a large value for the magnitude of the impulse is required to move the angle of approach that gives maximum variation of energy from $270^{\circ}$.

Fig. 5 and Table 4 show in more details the effects of the eccentricity of the primaries. The true anomaly of $M_{2}$ is fixed in $0^{\circ}$ and the angle of approach in $270^{\circ}$. The eccentricity is varied to assume the values $0.0,0.1,0.3$ and 0.5 . As explained before, it is very difficult to make predictions of the results. Even the rule of negative values for the direc- 


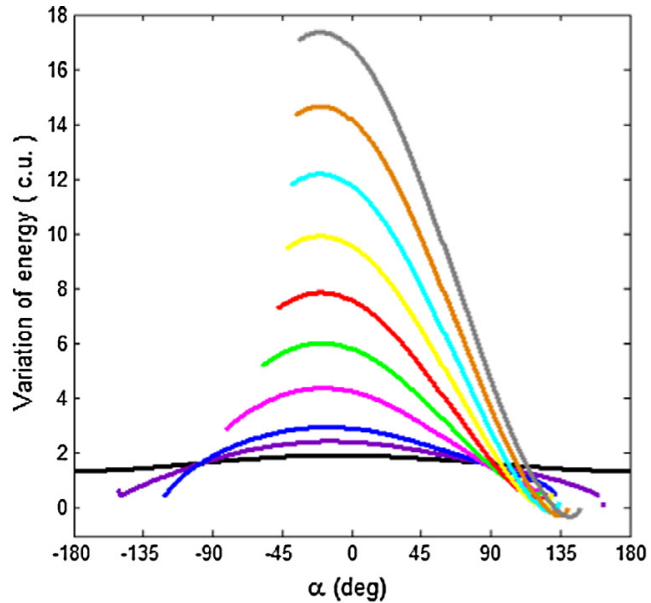

(a) $v=0^{\circ}$

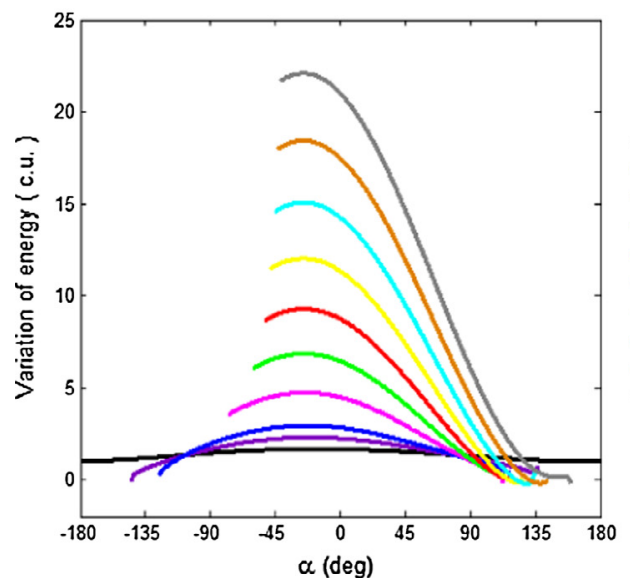

(c) $v=180^{\circ}$

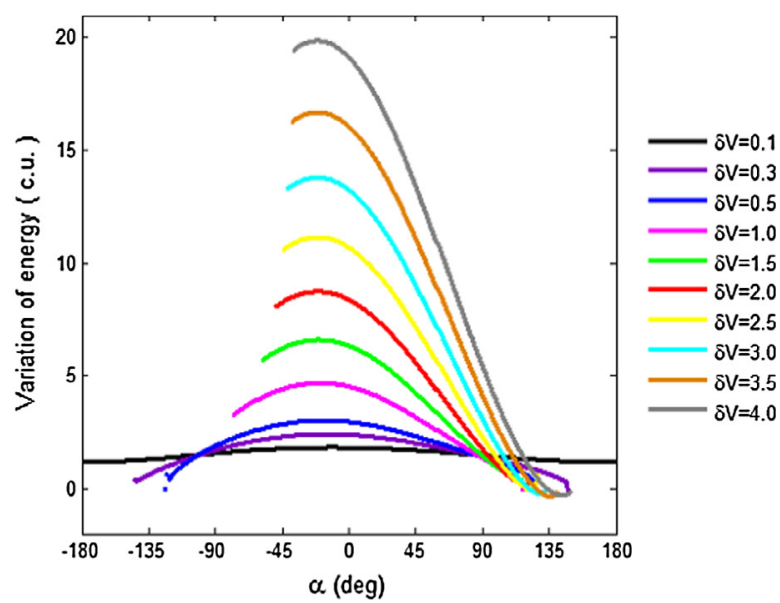

(b) $v=90^{\circ}$

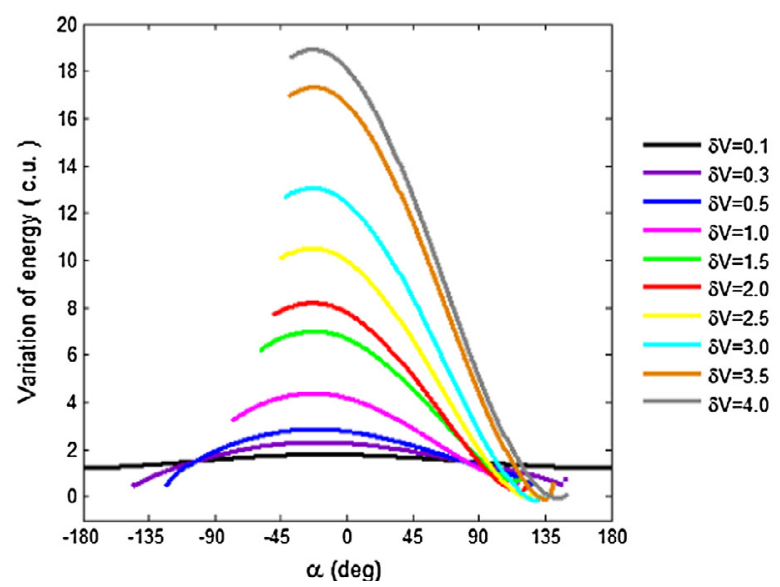

(d) $v=270^{\circ}$

Fig. 3. Variation of energy as a function of the direction of the impulse for $e=0.1$ and $\psi=270^{\circ}$.

Table 2

Maximum energy variation and the corresponding $\alpha$ for the case of Fig. $3\left(e=0.1\right.$ and $\left.\psi=270^{\circ}\right)$.

\begin{tabular}{|c|c|c|c|c|c|c|c|c|}
\hline \multirow[t]{2}{*}{$\delta V(\mathrm{~km} / \mathrm{s})$} & \multicolumn{2}{|l|}{$\underline{v}=0^{\circ}$} & \multicolumn{2}{|l|}{$\underline{v}=90^{\circ}$} & \multicolumn{2}{|l|}{$v=180^{\circ}$} & \multicolumn{2}{|l|}{$v=270^{\circ}$} \\
\hline & $\Delta E_{\max }$ (c.u.) & $\alpha\left({ }^{\circ}\right)$ & $\Delta E_{\max }$ (c.u.) & $\alpha\left({ }^{\circ}\right)$ & $\Delta E_{\max }$ (c.u.) & $\alpha\left(^{\circ}\right)$ & $\Delta E_{\max }$ (c.u.) & $\alpha\left({ }^{\circ}\right)$ \\
\hline 0.1 & 1.9248 & -9.0 & 1.8228 & -11.0 & 1.6809 & -17.0 & 1.7819 & -15.0 \\
\hline 0.3 & 2.4384 & -13.0 & 2.4197 & -15.0 & 2.2971 & -21.0 & 2.3040 & -19.0 \\
\hline 0.5 & 2.9611 & -16.0 & 3.0262 & -17.0 & 2.9385 & -22.0 & 2.8480 & -21.0 \\
\hline 1.0 & 4.3881 & -18.0 & 4.6835 & -19.0 & 4.7394 & -24.0 & 4.3722 & -23.0 \\
\hline 1.5 & 6.0203 & -19.0 & 6.5835 & -20.0 & 6.8516 & -25.0 & 7.0047 & -22.0 \\
\hline 2.0 & 7.8651 & -19.0 & 8.7361 & -21.0 & 9.279 & -25.0 & 8.1973 & -23.0 \\
\hline 2.5 & 9.9222 & -20.0 & 11.1417 & -21.0 & 12.0194 & -25.0 & 10.4976 & -23.0 \\
\hline 3.0 & 12.1901 & -20.0 & 13.7990 & -21.0 & 15.0706 & -25.0 & 13.0528 & -23.0 \\
\hline 3.5 & 14.6673 & -20.0 & 16.7068 & -21.0 & 18.4307 & -25.0 & 17.3258 & -22.0 \\
\hline 4.0 & 17.3524 & -20.0 & 19.8641 & -21.0 & 22.0982 & -25.0 & 18.9199 & -23.0 \\
\hline
\end{tabular}

tion of the impulse is no longer followed for the case $e=0.5$ and, for smaller values of the magnitude of the impulse, the values are positive. It is also noted that, for smaller values of the magnitude of the impulse, the expected rule of getting more variation of energy from the largest eccentricity is followed. This is due to the largest velocity of $M_{2}$ with respect to $M_{1}$. This rule does not hold for larger values of the impulse and the variations of energy reduce with the increase of the eccentricity. Remember that the goal is always to maximize the angle between the velocity vector of $M_{2}$ with respect to $M_{1}$ and the velocity of the spacecraft at the periapsis. 


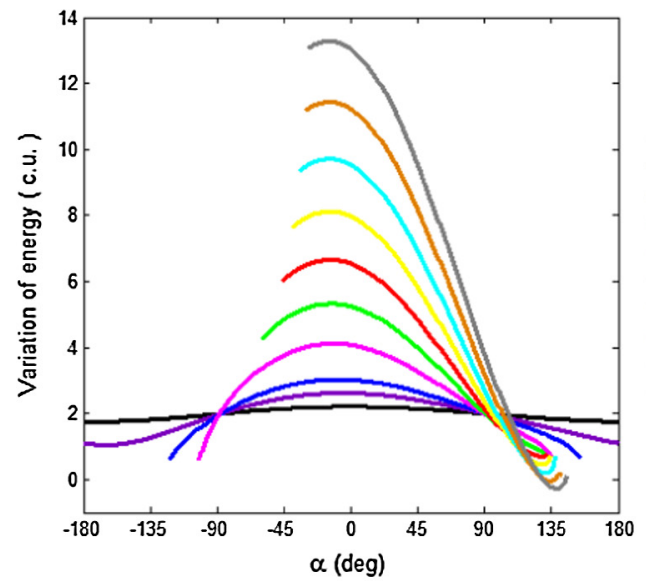

(a) $v=0^{\circ}$

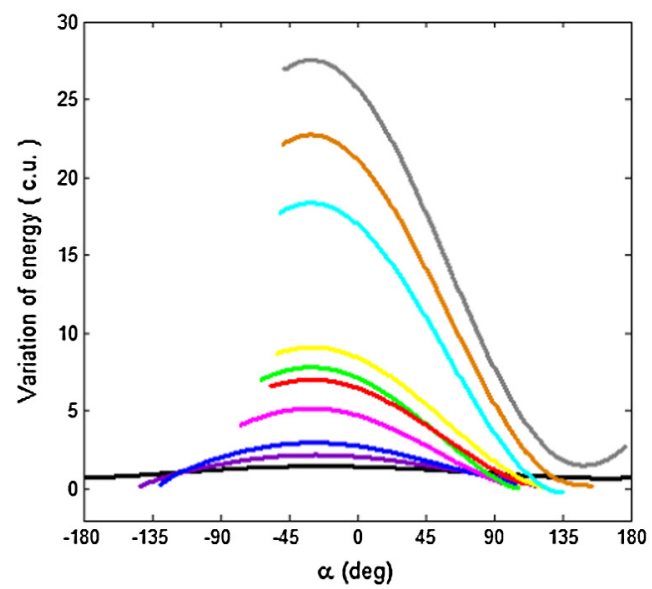

(c) $v=180^{\circ}$

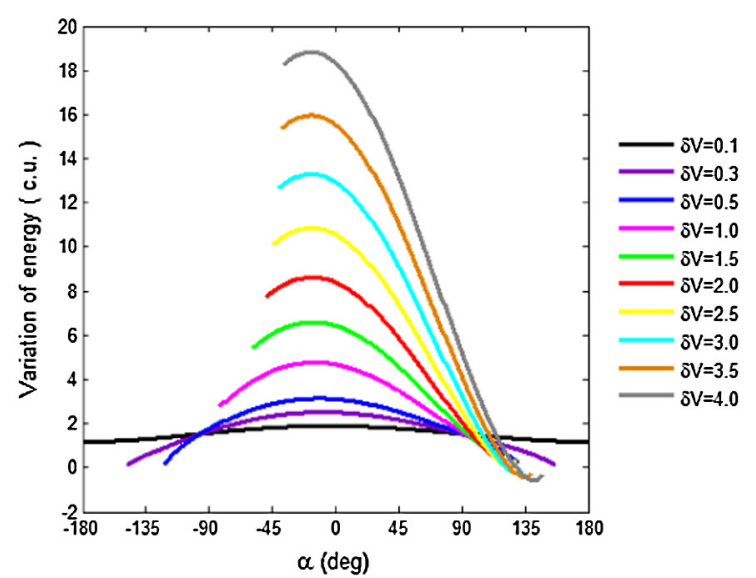

(b) $v=90^{\circ}$

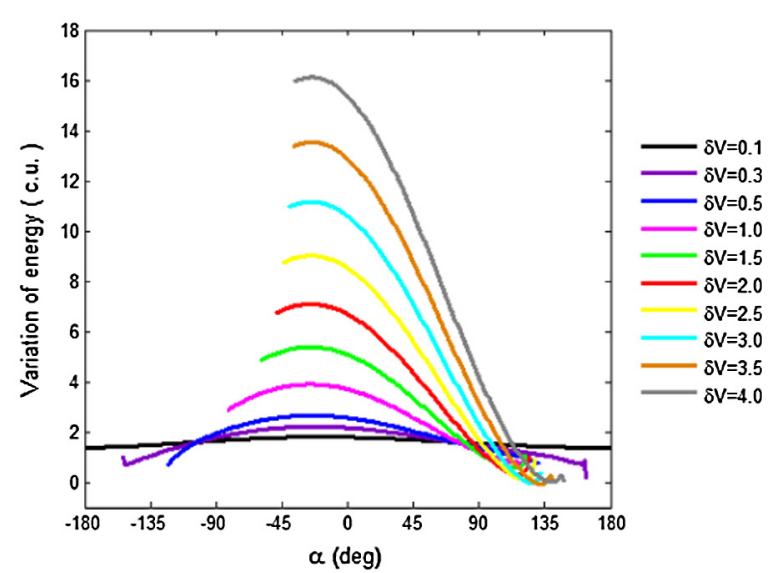

(d) $v=270^{\circ}$

Fig. 4. Variation of energy as a function of the direction of the impulse for $e=0.3$ and $\psi=270^{\circ}$.

Table 3

Maximum energy variation and the corresponding $\alpha$ for the case of Fig. $4\left(e=0.3\right.$ and $\left.\psi=270^{\circ}\right)$.

\begin{tabular}{|c|c|c|c|c|c|c|c|c|}
\hline \multirow[t]{2}{*}{$\delta V(\mathrm{~km} / \mathrm{s})$} & \multicolumn{2}{|l|}{$v=0^{\circ}$} & \multicolumn{2}{|l|}{$v=90^{\circ}$} & \multicolumn{2}{|l|}{$v=180^{\circ}$} & \multicolumn{2}{|l|}{$v=270^{\circ}$} \\
\hline & $\overline{\Delta E_{\max } \text { (c.u.) }}$ & $\alpha\left(^{\circ}\right)$ & $\Delta E_{\max }$ (c.u.) & $\alpha\left(^{\circ}\right)$ & $\Delta E_{\max }$ (c.u.) & $\alpha\left(^{\circ}\right)$ & $\Delta E_{\max }$ (c.u.) & $\alpha\left(^{\circ}\right)$ \\
\hline 0.1 & 2.2062 & -1.0 & 1.8895 & -5.0 & 1.4769 & -24.0 & 1.7946 & -18.0 \\
\hline 0.3 & 2.6180 & -5.0 & 2.5166 & -9.0 & 2.1985 & -27.0 & 2.2051 & -22.0 \\
\hline 0.5 & 3.0284 & -8.0 & 3.1360 & -11.0 & 2.9665 & -28.0 & 2.6427 & -24.0 \\
\hline 1.0 & 4.1148 & -11.0 & 4.7725 & -14.0 & 5.1751 & -29.0 & 3.8917 & -25.0 \\
\hline 1.5 & 5.3199 & -13.0 & 6.5944 & -15.0 & 7.8195 & -30.0 & 5.3718 & -25.0 \\
\hline 2.0 & 6.6537 & -13.0 & 8.6217 & -16.0 & 7.0127 & -29.0 & 7.0799 & -25.0 \\
\hline 2.5 & 8.1178 & -14.0 & 10.858 & -17.0 & 9.0900 & -30.0 & 9.0114 & -25.0 \\
\hline 3.0 & 9.7116 & -14.0 & 13.3049 & -17.0 & 18.3566 & -30.0 & 11.1627 & -24.0 \\
\hline 3.5 & 11.4342 & -14.0 & 15.9618 & -17.0 & 22.7282 & -30.0 & 13.531 & -24.0 \\
\hline 4.0 & 13.2847 & -14.0 & 18.8284 & -17.0 & 27.5265 & -30.0 & 16.1141 & -24.0 \\
\hline
\end{tabular}

The results also showed that the energy variation is directly proportional to the magnitude of the impulse in most cases. This conclusion is not applicable in some situations, for trajectories at the borders of the plots (relative to $x$-axis). It is due to the influence of $\alpha$ in those cases. This is the angle that defines the direction of the impulse. As shown in Fig. $1, \alpha$ is the angle between $\vec{V}_{p-}$ and $\overrightarrow{\delta V}$, where $\vec{V}_{p-}$ is the velocity of the spacecraft at periapsis before the powered maneuver. When the impulse is applied in the direction of motion of the spacecraft $\alpha$ is zero. The analysis of $\alpha$ can be divided in four parts: (i) $0^{\circ}<\alpha<90^{\circ}$ where the impulse has a component in the direction of motion of the spacecraft and another component in the direction opposite to $M_{2}$, making the spacecraft to move away from the body; (ii) $90^{\circ}<\alpha<180^{\circ}$, where the spacecraft also moves away from $M_{2}$, but the other component is opposite to the 


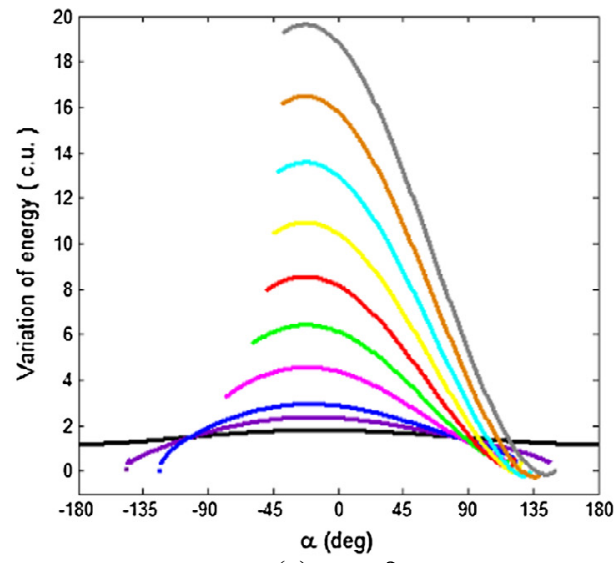

(a) $e=0$

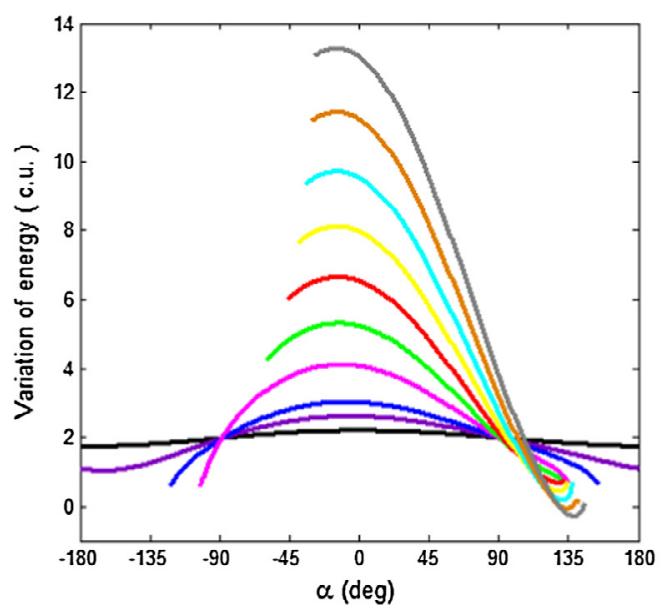

(c) $e=0.3$

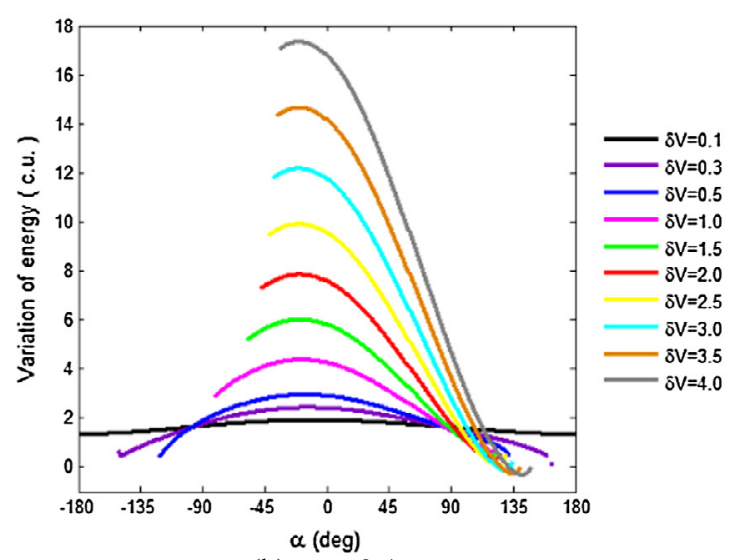

(b) $e=0.1$

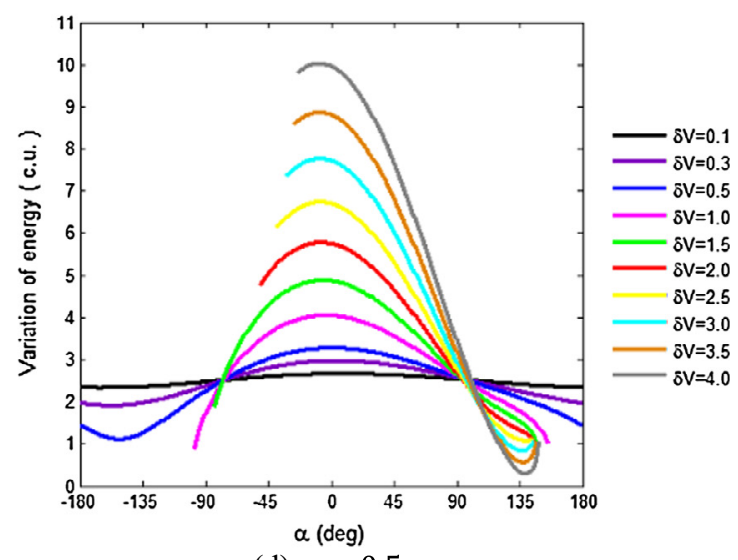

(d) $e=0.5$

Fig. 5. Variation of energy as a function of the direction of the impulse for $v=0^{\circ}$ and $\psi=270^{\circ}$.

Table 4

Maximum energy variation and the corresponding $\alpha$ for the case of Fig. $5\left(v=0^{\circ}\right.$ and $\left.\psi=270^{\circ}\right)$.

\begin{tabular}{|c|c|c|c|c|c|c|c|c|}
\hline \multirow[t]{2}{*}{$\delta V(\mathrm{~km} / \mathrm{s})$} & \multicolumn{2}{|l|}{$e=0$} & \multicolumn{2}{|l|}{$e=0.1$} & \multicolumn{2}{|l|}{$e=0.3$} & \multicolumn{2}{|l|}{$e=0.5$} \\
\hline & $\Delta E_{\max }$ (c.u.) & $\alpha\left({ }^{\circ}\right)$ & $\Delta E_{\max }$ (c.u.) & $\alpha\left({ }^{\circ}\right)$ & $\Delta E_{\max }$ (c.u.) & $\alpha\left(^{\circ}\right)$ & $\Delta E_{\max }$ (c.u.) & $\alpha\left(^{\circ}\right)$ \\
\hline 0.1 & 1.7971 & -13.0 & 1.9248 & -9.0 & 2.2062 & -1.0 & 2.6737 & 8.0 \\
\hline 0.3 & 2.3617 & -17.0 & 2.4384 & -13.0 & 2.618 & -5.0 & 2.9804 & 4.0 \\
\hline 0.5 & 2.9429 & -19.0 & 2.9611 & -16.0 & 3.0284 & -8.0 & 3.2821 & 1.0 \\
\hline 1.0 & 4.5526 & -21.0 & 4.3881 & -18.0 & 4.1148 & -11.0 & 4.057 & -4.0 \\
\hline 1.5 & 6.4183 & -22.0 & 6.0203 & -19.0 & 5.3199 & -13.0 & 4.8869 & -6.0 \\
\hline 2.0 & 8.5455 & -22.0 & 7.8651 & -19.0 & 6.6537 & -13.0 & 5.7814 & -7.0 \\
\hline 2.5 & 10.933 & -22.0 & 9.9222 & -20.0 & 8.1178 & -14.0 & 6.7428 & -8.0 \\
\hline 3.0 & 13.5788 & -22.0 & 12.1901 & -20.0 & 9.7116 & -14.0 & 7.7721 & -8.0 \\
\hline 3.5 & 16.4813 & -22.0 & 14.6673 & -20.0 & 11.4342 & -14.0 & 8.8687 & -8.0 \\
\hline 4.0 & 19.6391 & -22.0 & 17.3524 & -20.0 & 13.2847 & -14.0 & 10.0324 & -8.0 \\
\hline
\end{tabular}

motion of the spacecraft and it slow down its motion. This situation may result in captures or collisions; (iii) $-90^{\circ}<\alpha<0^{\circ}$, where the spacecraft tends to approach $M_{2}$ with a component in the direction of the secondary body and the other component in the direction of the motion of the spacecraft; and (iv) $-180^{\circ}<\alpha<-90^{\circ}$, where the spacecraft is slowed down by a component of the impulse against the motion of the spacecraft and the other component in the direction of $M_{2}$, which is the region most favorable for captures and collisions.

\subsection{Effects of the approach angle}

A more detailed study of the effects of the angle of approach $(\psi)$ can also be made. This angle defines the position of the periapsis with respect to the secondary body. 


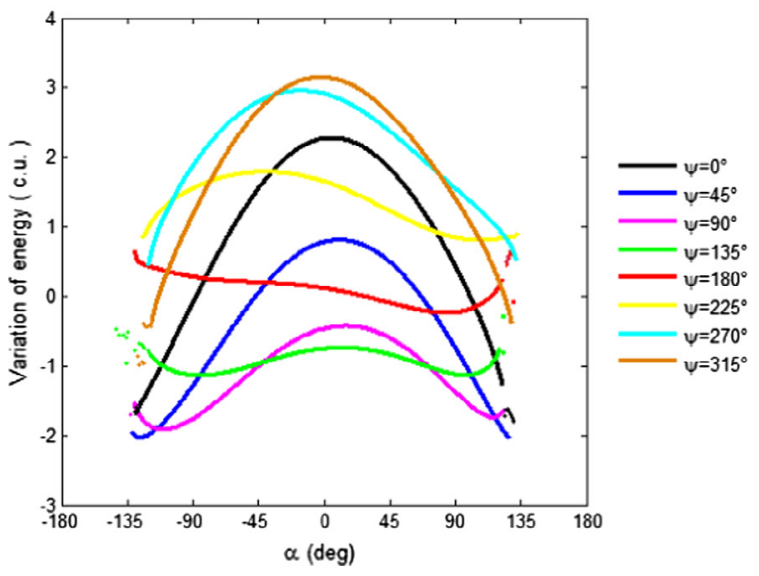

(a) $\delta V=0.5 \mathrm{~km} / \mathrm{s}$

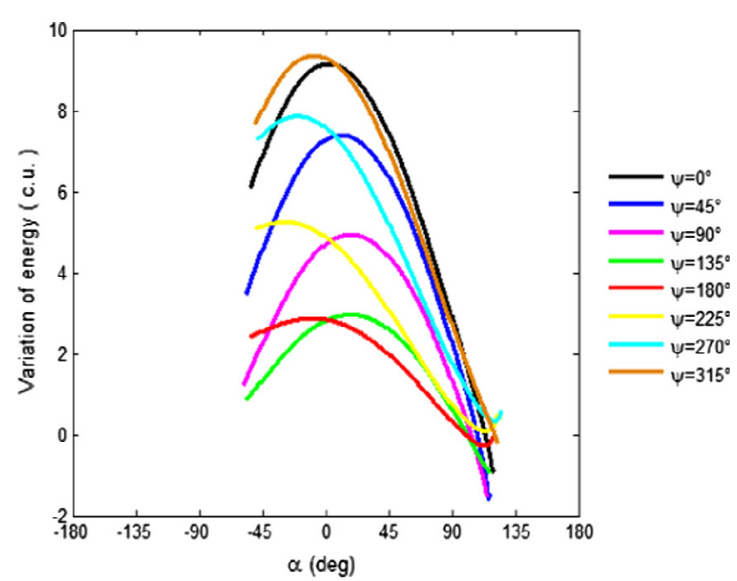

(b) $\delta V=2.0 \mathrm{~km} / \mathrm{s}$

Fig. 6. Energy variation for $e=0.1, v=270^{\circ}, \delta V=0.5$ and $\delta V=2.0 \mathrm{~km} / \mathrm{s}$ for different $\psi$.

When $0^{\circ}<\psi<180^{\circ}$ the spacecraft is passing in front of the secondary body, so it is decelerated by $M_{2}$ and it loses energy. The maximum loss occurs when $\psi=90^{\circ}$. If $180^{\circ}<\psi<360^{\circ}$, the spacecraft is passing behind $\mathrm{M}_{2}$ and it is accelerated, so gaining energy. The maximum energy gain occurs when $\psi=270^{\circ}$ For $\psi=0^{\circ}$ and $\psi=180^{\circ}$ the effect of the pure gravity maneuver is null. This conclusion comes from the equation that gives the energy variation for the standard Swing-By maneuver (Broucke, 1988). $E_{+}$is the energy of the second orbit; $E_{-}$the energy of the first orbit, $V_{2}$ the velocity of $M_{2}$ relative to $M_{1}$ and $\delta$ is the curvature of the trajectory of the spacecraft. The equation is (Broucke, 1988):

$E_{+}-E_{-}=-2 V_{i n f-} V_{2} \sin \delta \sin \psi$

Fig. 6 and Table 5 show some results for the "Powered Swing-By Maneuver". They confirm that the angle of approach plays an important role in the energy gains. For a maneuver in the circular case with no impulse, the case $\psi=270^{\circ}$ would give the maximum variation of energy. But, it is important to remember that the impulse and the eccentricity of the primaries change the real value of the angle of approach. The numerical results show that the case $\psi=315^{\circ}$ has some points with higher variation of energy. It happens because this combination makes the real angle of approach to get closer to the maximum point $\left(\psi=270^{\circ}\right)$, while orbits with $\psi=270^{\circ}$ are deviated from

Table 5

Maximum energy variation and the corresponding $\alpha$ for the case of Fig. 6 $\left(e=0.1\right.$ and $\left.v=0^{\circ}\right)$.

\begin{tabular}{llllll}
\hline$\psi\left({ }^{\circ}\right)$ & \multicolumn{2}{l}{$\delta V=0.5 \mathrm{~km} / \mathrm{s}$} & & \multicolumn{2}{l}{$\delta V=2.0 \mathrm{~km} / \mathrm{s}$} \\
\cline { 2 - 3 } \cline { 6 - 6 } & $\Delta E_{\max }$ (c.u.) & $\alpha\left(^{\circ}\right)$ & & $\Delta E_{\max }($ c.u. $)$ & $\alpha\left(^{\circ}\right)$ \\
\hline 0 & 2.2784 & 6.0 & 9.1525 & 13.0 \\
45 & 0.8131 & 11.0 & 7.3981 & 11.0 \\
90 & -0.4206 & 15.0 & 4.9422 & 18.0 \\
135 & -0.0914 & -132.0 & 2.9641 & 18.0 \\
180 & 0.7087 & 129.0 & 2.8683 & -9.0 \\
225 & 1.7953 & -39.0 & 5.2489 & -29.0 \\
270 & 2.9611 & -16.0 & 7.8651 & -19.0 \\
315 & 3.1518 & -2.0 & 9.3545 & -8.0 \\
\hline
\end{tabular}

this maximum point by the impulse. It is also noted that the case $\psi=0^{\circ}$ has larger variations of energy, because it is a case where the impulse modifies the trajectory and put the angle of approach in the region of gains of energy, as explained before. It is also noted that the minimum variation of energy did not occur exactly at $\psi=90^{\circ}$, also due to the modification of the angle of approach by the impulse. These facts emphasize the importance of the present study, because only the numerical solutions can find the regions of maximum and minimum variations of energy in this more complex maneuver.

\subsection{Effects of the eccentricity}

The physical effect of the eccentricity is to increase the velocity of $M_{2}$ with respect to the center of mass of the system, since it is no longer constant. $V_{2}$ is larger when $M_{2}$ is at periapsis and smaller when it is in the apoapsis of the orbit. As shown by Eq. (4), the variation of energy of the spacecraft is proportional to $V_{2}$. The results are shown for fixed values of $v, \delta V$ and $\psi$. Fig. 7 shows the spacecraft energy variation for $M_{2}$ at the periapsis of the orbit $\left(v=0^{\circ}\right)$, with angle of approach $\psi=90^{\circ}$, and two values for the magnitude of the impulse: $\delta V=0.1 \mathrm{~km} / \mathrm{s}$ and $\delta V=2.0 \mathrm{~km} / \mathrm{s}$. This is the situation with loss of energy, so the main effect of increasing the eccentricity is to get larger losses of energy. The peaks for every line are near zero, with respect to the direction of the impulse. There is a very uniform behavior for $\delta V=0.1 \mathrm{~km} / \mathrm{s}$. The case with $\delta V=2.0 \mathrm{~km} / \mathrm{s}$ results in stronger effects in the geometry and the results are less uniform and less predictable, as explained before. It is also noted the appearances of captures and collisions, since now the impulse is capable of making stronger modifications in the orbit of the spacecraft (see Table 6).

\subsection{Effects of the true anomaly of $M_{2}(v)$}

The true anomaly of $M_{2}(v)$ describes the position of the secondary body with respect to $M_{1}$. For $v=0^{\circ}, M_{2}$ is at the 


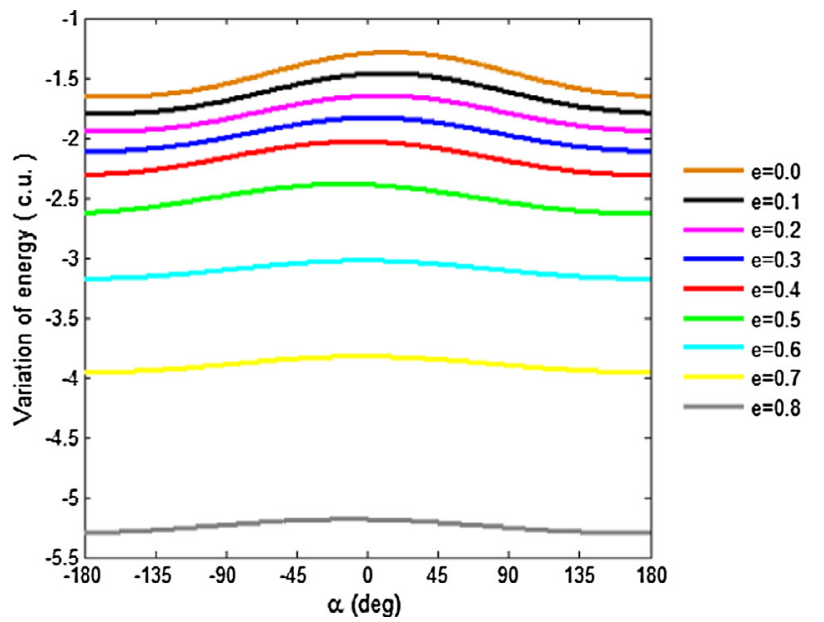

(a) $\delta V=0.1 \mathrm{~km} / \mathrm{s}$

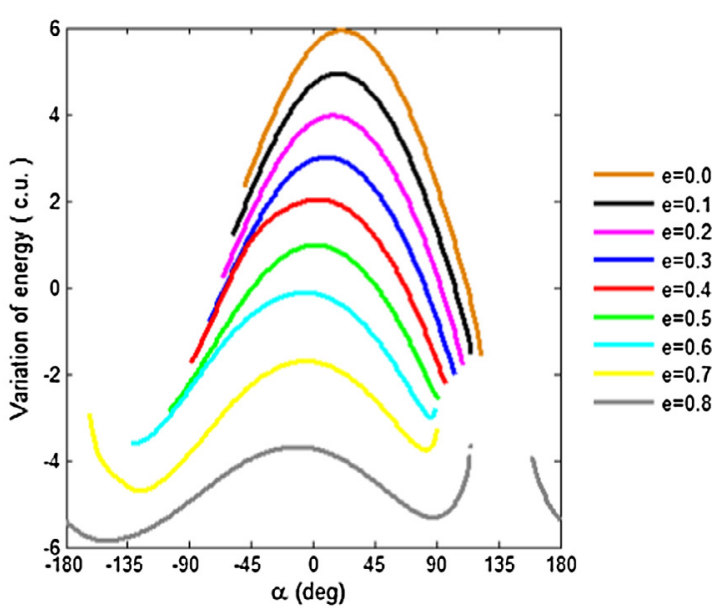

(b) $\delta V=2.0 \mathrm{~km} / \mathrm{s}$

Fig. 7. Energy variation for $v=0^{\circ}, \psi=90^{\circ}$ and different eccentricity.

Table 6

Maximum energy variation and the corresponding $\alpha$ for the case of Fig. 7 $\left(v=0^{\circ}\right.$ and $\left.\psi=90^{\circ}\right)$.

\begin{tabular}{llllll}
\hline$e$ & \multicolumn{2}{l}{$\delta V=0.1 \mathrm{~km} / \mathrm{s}$} & & \multicolumn{2}{l}{$\delta V=2.0 \mathrm{~km} / \mathrm{s}$} \\
\cline { 2 - 3 } \cline { 6 - 6 } & $\Delta E_{\max }($ c.u. $)$ & $\alpha\left(^{\circ}\right)$ & & $\Delta E_{\max }($ c.u. $)$ & $\alpha\left(^{\circ}\right)$ \\
\hline 0.0 & -1.2852 & 16.0 & 5.9348 & 21.0 \\
0.1 & -1.4616 & 13.0 & 4.9422 & 18.0 \\
0.2 & -1.6466 & 9.0 & 3.9697 & 15.0 \\
0.3 & -1.8323 & 5.0 & 3.0127 & 10.0 \\
0.4 & -2.0313 & -6.0 & 2.0351 & 4.0 \\
0.5 & -2.3872 & -15.0 & 4.3606 & -78.0 \\
0.6 & -3.0241 & 0.0 & -0.3050 & 0.0 \\
0.7 & -3.8249 & -2.0 & -1.7057 & -5.0 \\
0.8 & -5.1829 & -12.0 & -3.6217 & 114.0 \\
\hline
\end{tabular}

periapsis and, for $v=180^{\circ}, M_{2}$ is at the apoapsis. Therefore, $v$ influences the magnitude and direction of the velocity of $M_{2}\left(V_{2}\right)$, which then influences the variation of energy. For this study $v$ assumed the values $0^{\circ}, 90^{\circ}, 180^{\circ}$ and $270^{\circ}$. The results are shown in Fig. 8 and Table 7 .
For $\delta V=0.3 \mathrm{~km} / \mathrm{s}$, the variations of energy follows the expected behavior of having the maximum variations of energy when $M_{2}$ is at the periapsis, because the velocity of $M_{2}$ is larger, and the minimum variations occur when $M_{2}$ is at the apoapsis. For $\delta V=1.5 \mathrm{~km} / \mathrm{s}$ the powered part of the maneuver dominates the scenario and the best value for the true anomaly cannot be predicted without the numerical analysis. In most cases the position where $M_{2}$ is at the periapsis has even the minimum variation of energy. The final results come from a combination of all of the conditions discussed above.

\subsection{Captures and collisions}

There are several maneuvers ending in captures or collisions of the spacecraft with $M_{2}$. A capture is a situation where the spacecraft remains around the secondary body with negative two body energy during the total integration period. A collision is when the spacecraft crash with the secondary body.

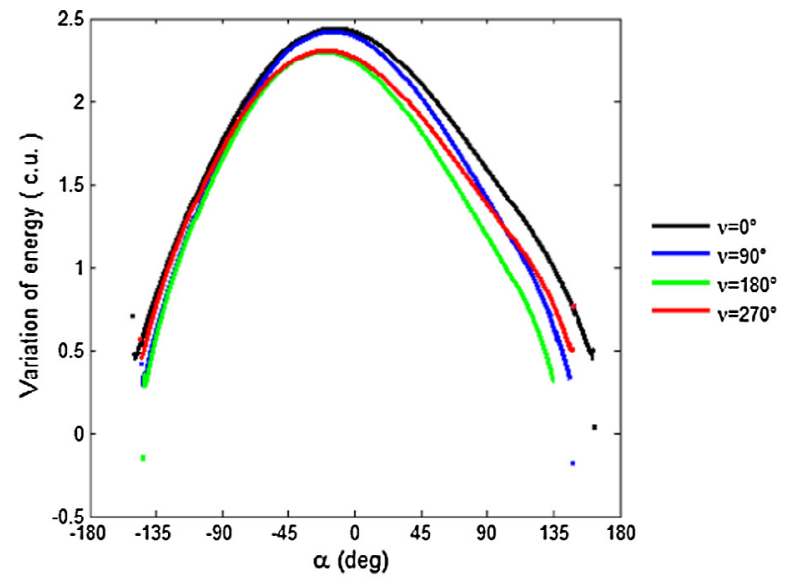

(a) $\delta V=0.3 \mathrm{~km} / \mathrm{s}$

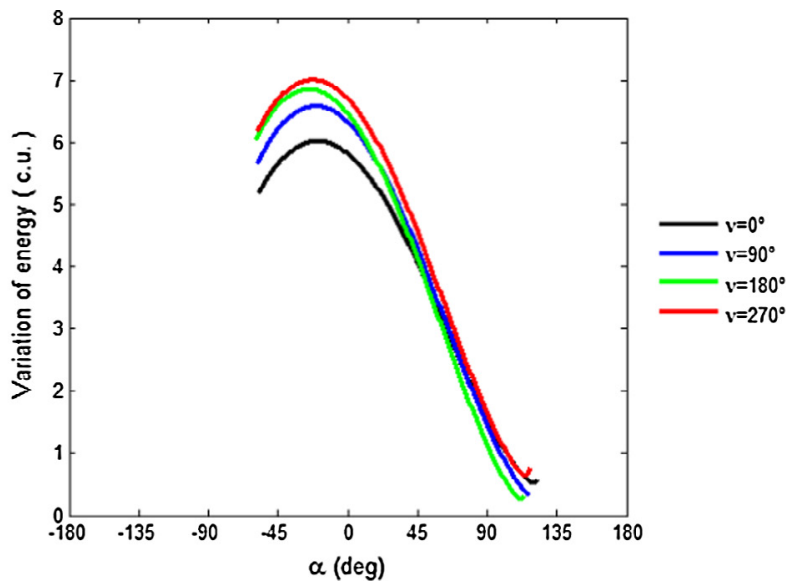

(b) $\delta V=1.5 \mathrm{~km} / \mathrm{s}$

Fig. 8. Energy variation for $e=0.1, \psi=270^{\circ}$, and different true anomalies of $M_{2}$. 
Table 7

Maximum energy variation and the corresponding $\alpha$ for the cases shown in Fig. $8\left(e=0.1\right.$ and $\left.\psi=270^{\circ}\right)$.

\begin{tabular}{llllll}
\hline$v\left(^{\circ}\right)$ & \multicolumn{2}{l}{$\delta V=0.3 \mathrm{~km} / \mathrm{s}$} & & \multicolumn{2}{l}{$\delta V=1.5 \mathrm{~km} / \mathrm{s}$} \\
\cline { 2 - 3 } \cline { 6 - 6 } & $\Delta E_{\max }$ (c.u.) & $\alpha\left(^{\circ}\right)$ & & $\Delta E_{\max }$ (c.u.) & $\alpha\left(^{\circ}\right)$ \\
\hline 0 & 2.4384 & -13.0 & 6.0203 & -19.0 \\
90 & 2.4197 & -15.0 & 6.5835 & -20.0 \\
180 & 2.2971 & -21.0 & 6.8516 & -25.0 \\
270 & 2.3040 & -19.0 & & 7.0047 & -22.0 \\
\hline
\end{tabular}

In most cases the captures and collisions occurred for values of $\alpha$ near the border of the plots. It happens because those values correspond to situations where the component of the impulse decelerates the spacecraft, acting in the direction opposite to the motion of the spacecraft. For situations where there is also a component sending the spacecraft to the direction of $M_{2}$, the amount of captures and collisions are significantly higher. For all cases, when $\delta V=0.1 \mathrm{~km} / \mathrm{s}$, there were no captures and collisions, because this magnitude of the impulse is not large enough to generate those situations. Next, some trajectories resulting in collisions are shown. The black solid line represents the trajectory of the spacecraft and the red line represents the surface of $M_{2}$.

Fig. 9 shows one trajectory ending in collision for $=0.1$, $v=0^{\circ}, \psi=0^{\circ}, \delta V=0.3 \mathrm{~km} / \mathrm{s}$ and $\alpha=144^{\circ}$. The spacecraft leaves $M_{2}$ due to the positive value of $\alpha$, it is decelerated, and then it returns back to collide with $M_{2}$.

Fig. 10 shows one trajectory colliding with $M_{2}$ for $e=0.1, v=0^{\circ}, \psi=270^{\circ}, \delta V=0.5 \mathrm{~km} / \mathrm{s}$ and $\alpha=-156^{\circ}$. In this case there was a temporary capture before the collision. The black solid line is the trajectory of the spacecraft and the red line the surface of $M_{2}$.

Next, Figs. 11 and 12 show trajectories resulting in the capture of the spacecraft by $M_{2}$. The trajectory shown in Fig. 11 has an impulse with component in the opposite

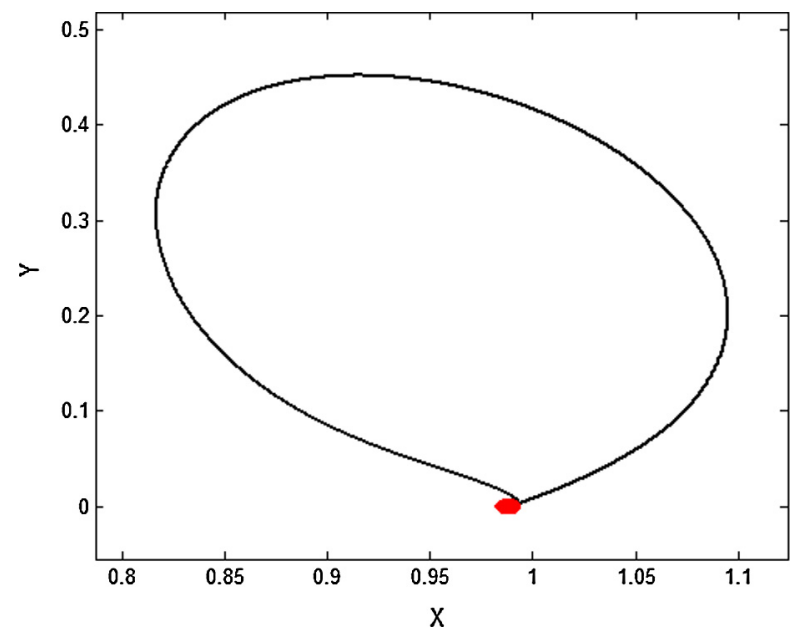

Fig. 9. Spacecraft trajectory resulting in collision for $e=0.1, v=0^{\circ}$, $\psi=0^{\circ}, \delta V=0.3 \mathrm{~km} / \mathrm{s}$ and $\alpha=144^{\circ}$.

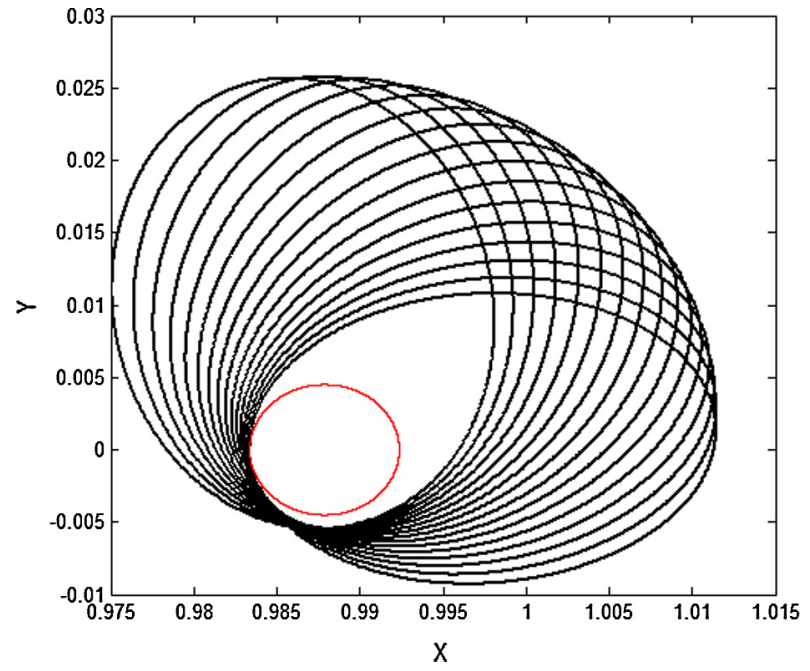

Fig. 10. Spacecraft trajectory resulting in collision for $e=0.1, v=0^{\circ}$, $\psi=270^{\circ}, \delta V=0.5 \mathrm{~km} / \mathrm{s}$ and $\alpha=-156^{\circ}$.

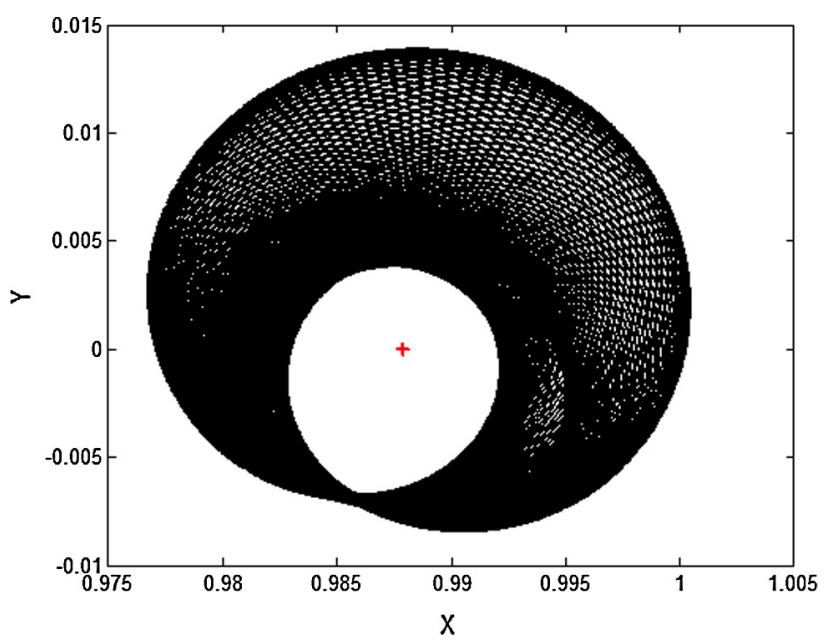

Fig. 11. Spacecraft trajectory resulting in capture for $e=0.3, v=180^{\circ}$, $\psi=90^{\circ}, \delta V=0.5 \mathrm{~km} / \mathrm{s}$ and $\alpha=-180^{\circ}$.

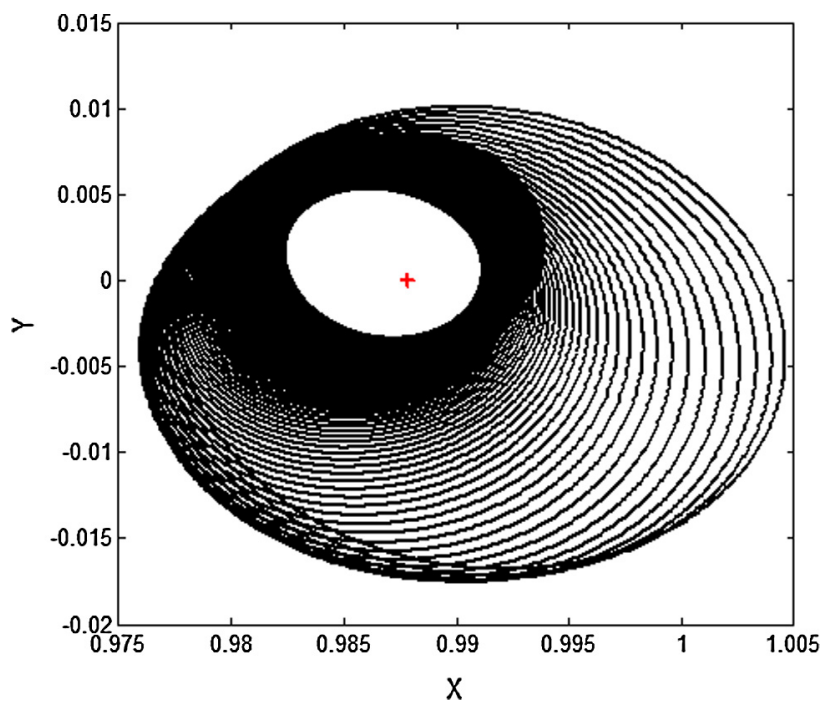

Fig. 12. Spacecraft trajectory resulting in capture for $e=0.5, v=0^{\circ}$, $\psi=180^{\circ}, \delta V=1.5 \mathrm{~km} / \mathrm{s}$ and $\alpha=155^{\circ}$. 
direction of the motion of the spacecraft, which reduces its velocity and causes the capture. The data are: $e=0.3$, $v=180^{\circ}, \psi=90^{\circ}, \delta V=0.5 \mathrm{~km} / \mathrm{s}$ and $\alpha=-180^{\circ}$. Fig. 12 shows the trajectory for $e=0.5, v=0^{\circ}, \quad \psi=180^{\circ}$, $\delta V=1.5 \mathrm{~km} / \mathrm{s}$ and $\alpha=155^{\circ}$, which is similar to the previous one, but that appears in a situation of larger eccentricity of the primaries $(e=0.5)$.

\subsection{Maximum energy variations}

After all the results and explanations made up to now, some plots show the maximum variations of energy and the respective direction of the impulse as a function of the angle of approach, for different eccentricities of the primaries. The true anomaly of $M_{2}$ at the moment of the closest approach was varied to consider the same values used before: $v=0^{\circ}, v=90^{\circ}, v=180^{\circ}, v=270^{\circ}$. The magnitude of the impulse assumed the values $0.1,0.3,0.5,1.0,2.0,2.5$, $3.0,3.5,4.0 \mathrm{~km} / \mathrm{s}$. The results are shown in Figs. $13-15$ for the situations where $e=0.1, e=0.3$ and $e=0.5$, respectively. The idea is to summarize all the analyses made up to this point.
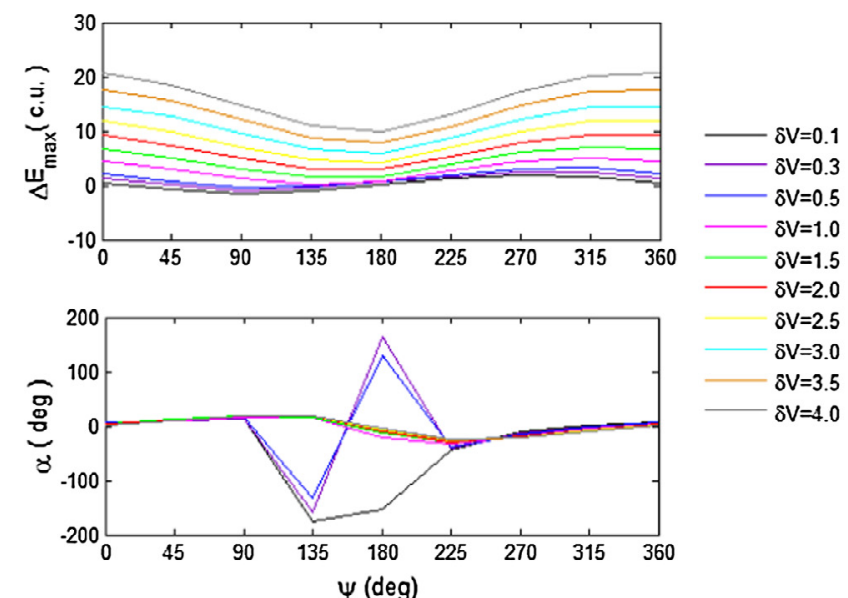

(a) $v=0^{\circ}$
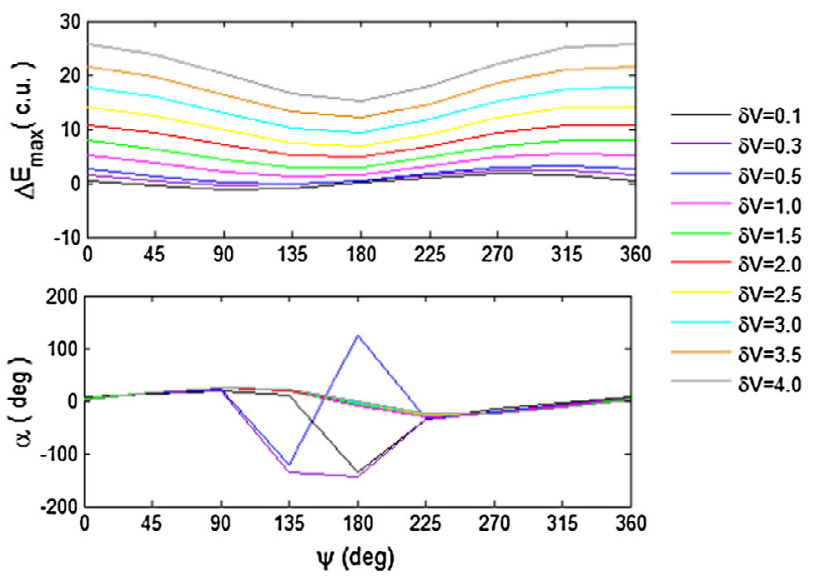

(c) $v=180^{\circ}$
In that sense, the variations of energy shown in Fig. 13 $(e=0.1)$ show in some more detail some results already noted and explained. It quantifies how much the variation of energy increases with the magnitude of the impulse. For smaller values for the magnitude of the impulse, the curve follows the results expected from the "patched conics pure gravity" maneuver (Broucke, 1988) and the maximum variations of energy is located in $\psi=270^{\circ}$, the minimum in $\psi=90^{\circ}$, and the near zero values in $\psi=0^{\circ}$, and $\psi=180^{\circ}$. The increase of the impulse shift those results and now the maximum variations of energy are located in $\psi=0^{\circ}$, and $\psi=360^{\circ}$, with the minimum in $\psi=180^{\circ}$. The reason is the shift in the real value of the angle of approach made by the impulse, as well as the periapsis distance, which gets the best combination of the gains of energy coming from the gravity and the powered parts of the maneuver. This is valid for all the true anomalies used, but the values of the variations of energy are affected by the true anomaly. The direction of the impulse remains near zero most of the times, with some exceptions in cases where a retrograde impulse is required to increase the gains obtained from the gravity part of the maneuver.

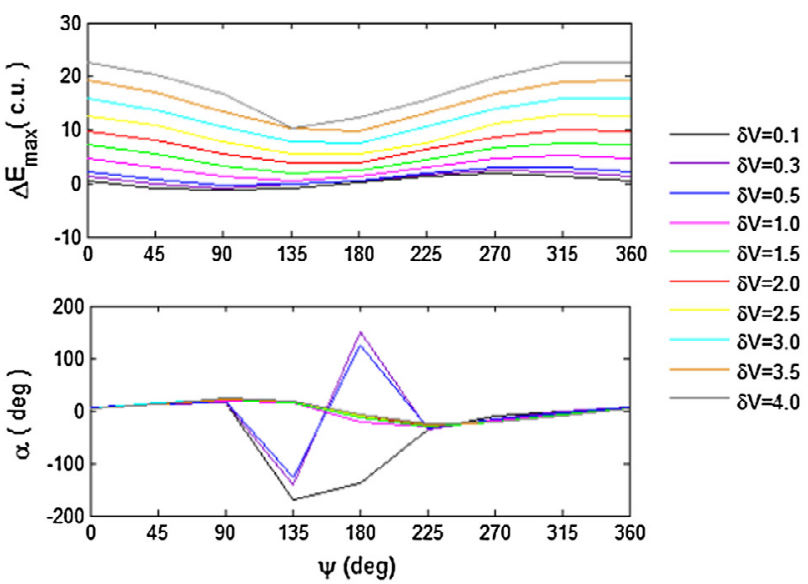

(b) $v=90^{\circ}$
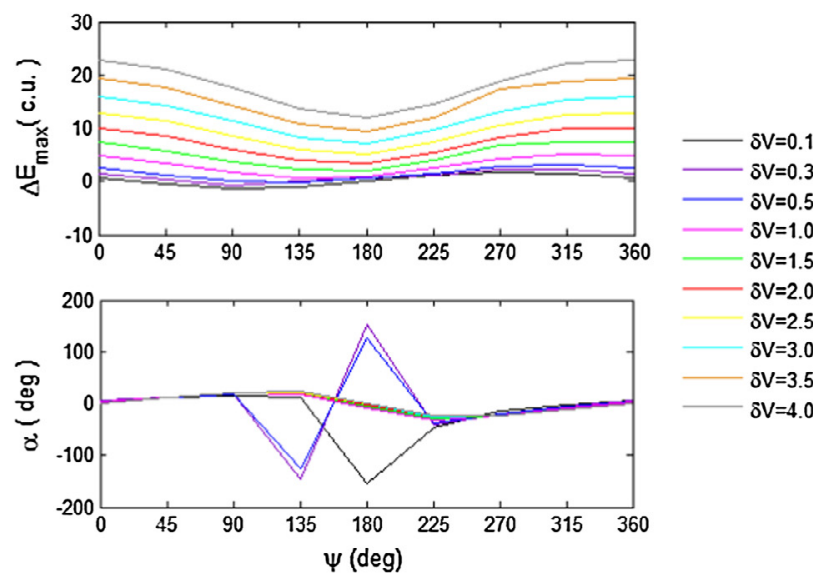

(d) $v=270^{\circ}$

Fig. 13. Maximum variation of energy and direction of the impulse vs. angle of approach for $e=0.1$. 


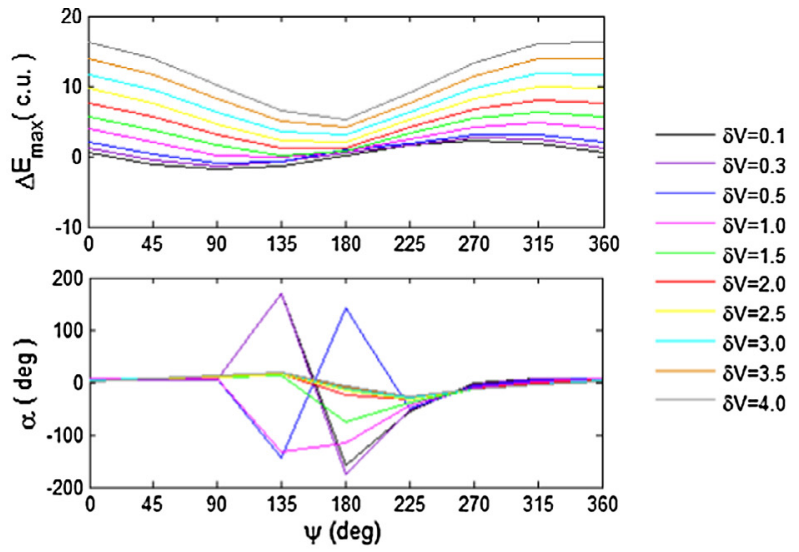

(a) $v=0^{\circ}$

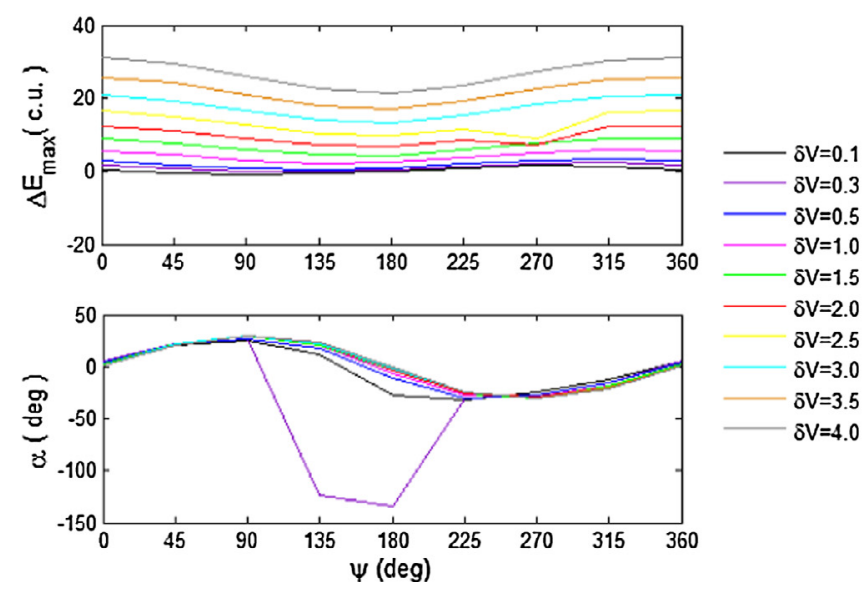

(c) $v=180^{\circ}$

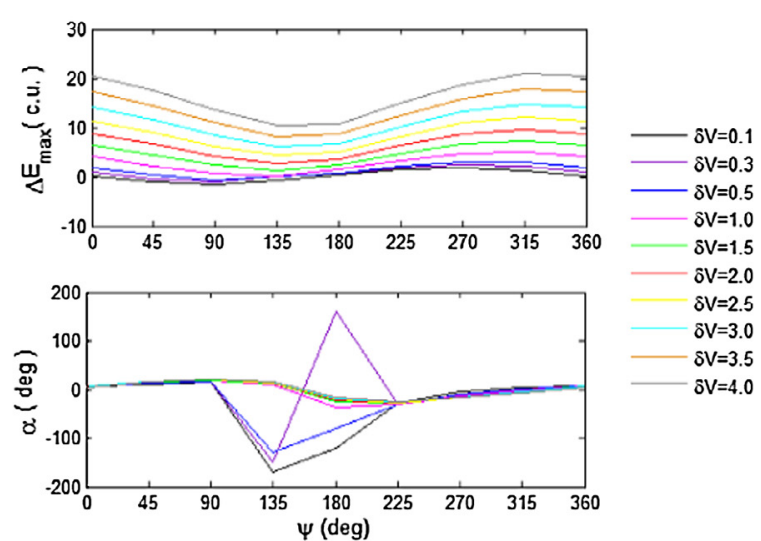

(b) $v=90^{\circ}$

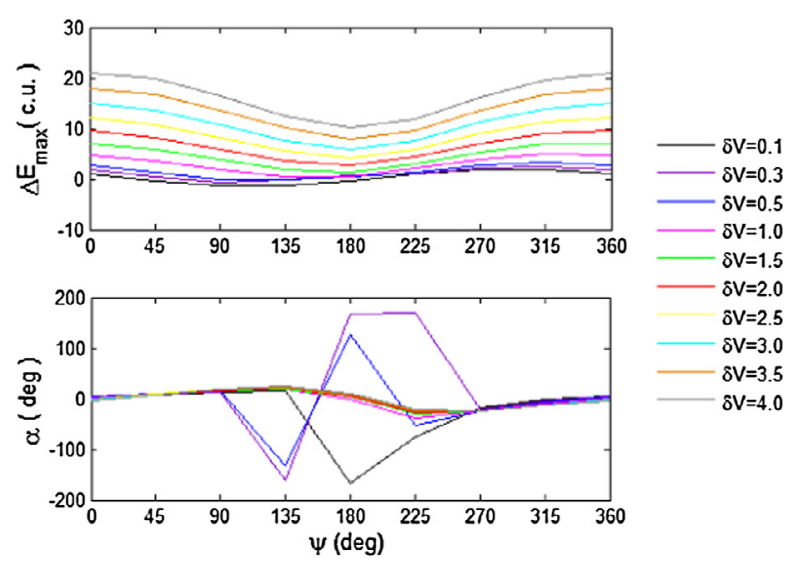

(d) $v=270^{\circ}$

Fig. 14. Maximum variation of energy and direction of the impulse vs. angle of approach for $e=0.3$.

The variations of energy shown in Fig. $14(e=0.3)$ show the same characteristics observed in Fig. 13. The main difference is an increase in the magnitude of the variations of energy, obtained from the increase of the velocity of $M_{2}$ around $M_{1}$. The importance of the true anomaly is also increased, for the same reason.

Fig. 15 shows the variations of energy for $e=0.5$. The case where $v=0^{\circ}$ shows a regular behavior for the maximum energy variations. For $\psi=180^{\circ}$ the magnitudes of the maximum energy variations remains very similar, for different values of $\delta V$, compared with the other values of $\psi$. It shows a regular behavior for the energy variations, similar to the results obtained in the previous cases. The direction of the impulse has also a regular behavior, having the form of a sin function. The directions of the impulse cover a larger range of results for $\psi$ between $90^{\circ}$ and $270^{\circ}$. The case where $v=90^{\circ}$ shows a sharp minimum variation of energy for $\psi=225^{\circ}$ for larger values of the impulse, with the maximum again near $\psi=0^{\circ}$ and $\psi=360^{\circ}$. This pattern is true even for situations with small values for the magnitude of the impulse.
The trajectory resulting in the maximum variation of energy occurs when $M_{2}$ is at the periapsis of its orbit and the angle of approach is $\psi=0^{\circ}$, for $\delta V=4.0 \mathrm{~km} / \mathrm{s}$, for the eccentricities shown in Fig. 16. The dashed lines represents the first part of the orbit (before the impulse) and the continuous lines the second part (after impulse). The trajectory (a) is the natural path of the spacecraft in the circular problem without the impulsive maneuver. It is shown for comparisons. The (b)-(d) trajectories refer to the eccentricities $0.1,0.3$ and 0.5 , respectively.

\section{Conclusions}

The energy variations in a powered Swing-By maneuver in the situation where the primaries of the system are in elliptical orbits were studied. The inclusion of the eccentricity of the primaries gives a more realistic approach to the problem, in particular if the maneuver is performed in systems of high eccentricity. It also gives more options to get more energy from the maneuver, by exploring the variations of the velocity of $M_{2}$. 


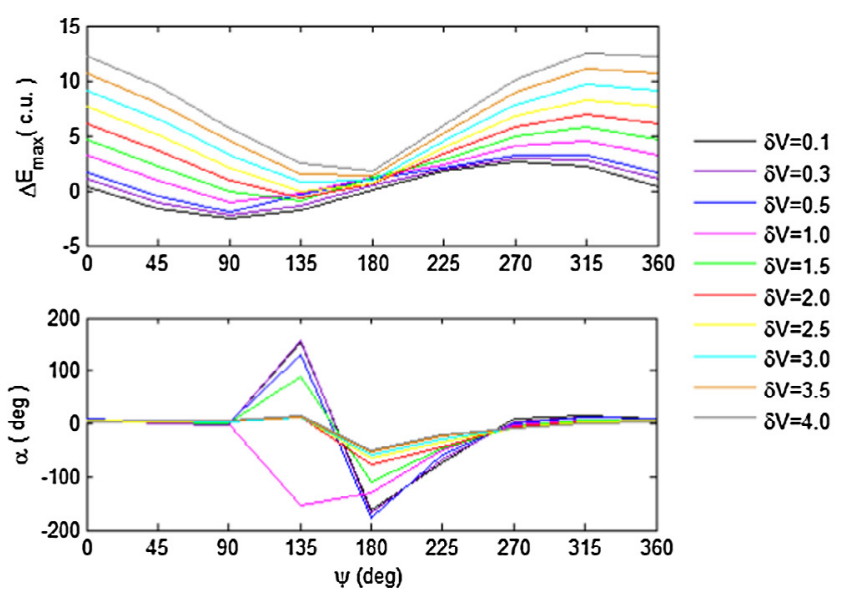

(a) $v=0^{\circ}$

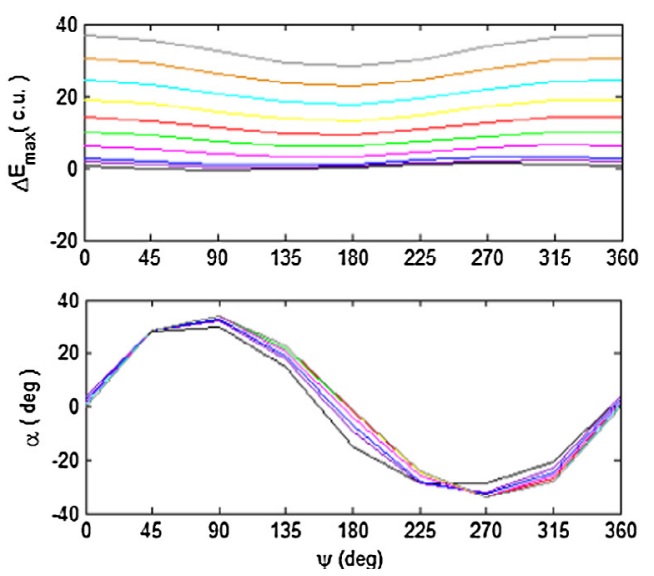

(c) $v=180^{\circ}$

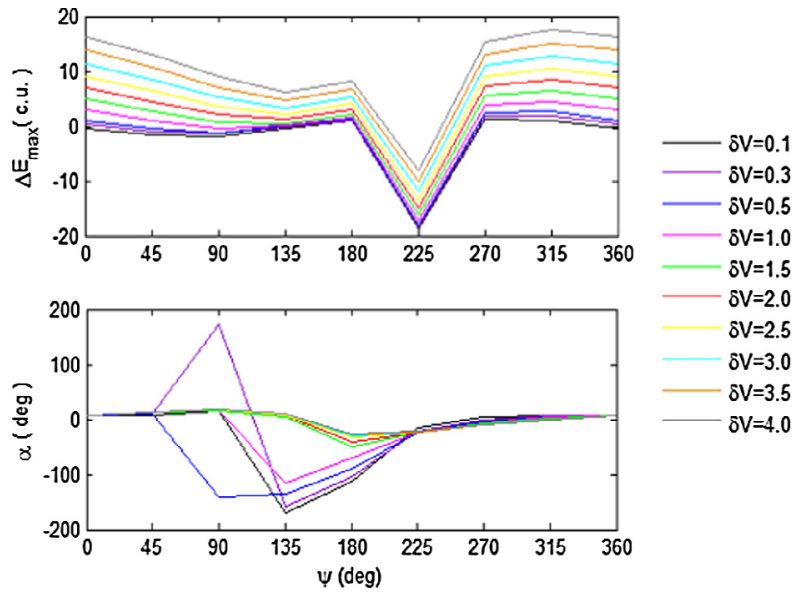

(b) $v=90^{\circ}$

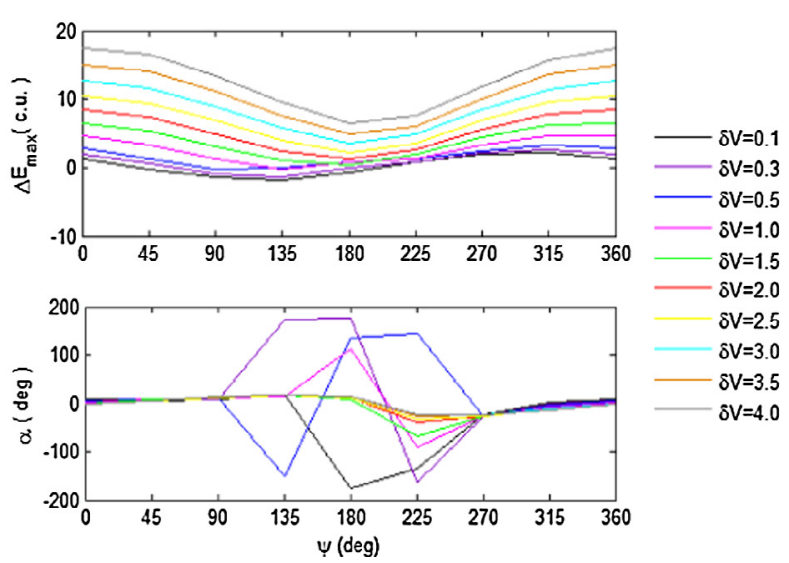

(d) $v=270^{\circ}$

Fig. 15. Maximum variation of energy and direction of the impulse vs. angle of approach for $e=0.5$.

These variations of energy can be optimized according to the needs of the mission and the best direction to apply the impulse can be found as a function of the magnitude of the impulse available to the spacecraft.

The initial conditions are the parameters that define the orbit of the primaries $(e, v)$, the orbit of the spacecraft $\left(V_{\text {inf }-}, r_{p}, \psi\right)$ and the features of the impulse $(\delta V, \alpha)$. The study concentrated in measuring the effects of each one of these parameters.

The main physical modification made by the inclusion of the eccentricity $(e)$ is the variation of the velocity $\left(\mathrm{V}_{2}\right)$ of $M_{2}$ with respect to $M_{2}$, which is constant in the circular problem. It now depends on the position of $M_{2}$, which is defined by its true anomaly $(v)$. The gain of energy coming from the gravity part of the maneuver is dependent on $V_{2}$.

The results showed that applying the impulse in a nontangential direction optimizes the maneuver, getting more energy from the powered maneuver. The largest gain of energy comes from a better combination of the modifications made by the impulse in the geometry of the trajectory, which changes the angle of approach and the periapsis distance, to get more energy from the gravity part of the maneuver, with the gains obtained directly from the application of the impulse.

Maneuvers with small values for the magnitude of the impulse has more predictable results, following the rules made by the "patched conics pure gravity assisted maneuver". With the increase of the magnitude of the impulse only a numerical algorithm can give the best direction to apply the impulse as a function of the other parameters, which justify the study made in the present paper.

The present study can help the planning of missions including powered swing-by maneuvers, in particular with high eccentricities system of primaries, by indicating the best directions to apply the impulse in several situations. 


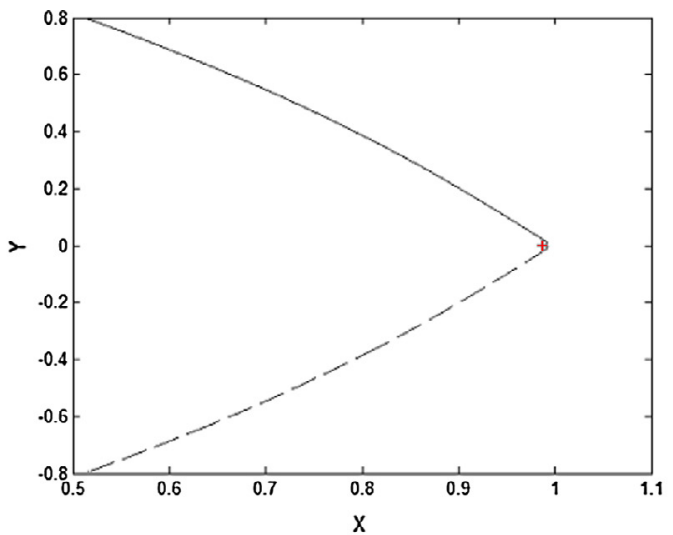

(a) $e=0$

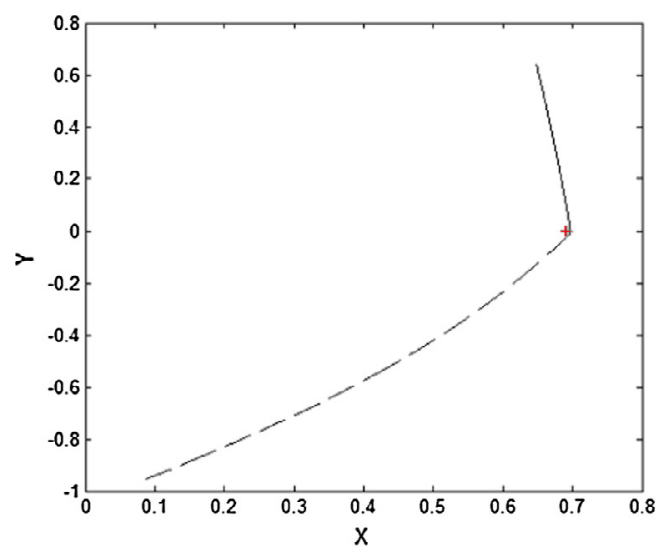

(c) $e=0.3$

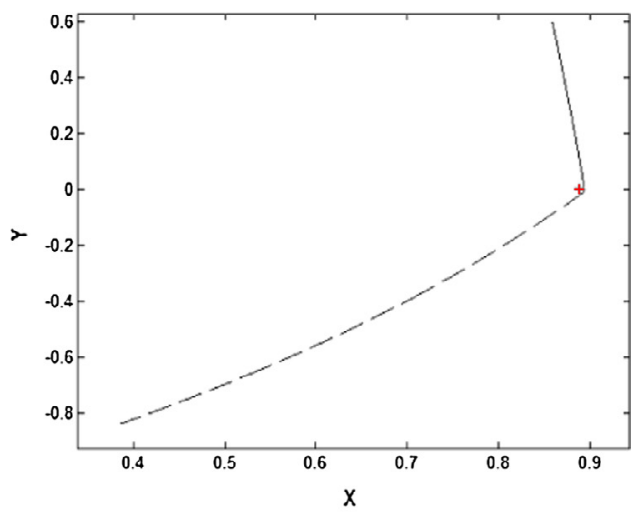

(b) $e=0.1$

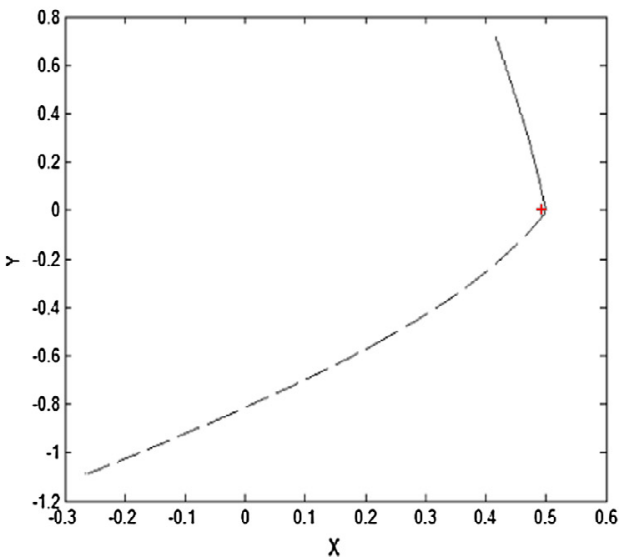

(d) $e=0.5$

Fig. 16. Trajectories with conditions resulting in maximum variations of energy for $v=0^{\circ}, \psi=0^{\circ}$ and $\delta V=4.0 \mathrm{~km} / \mathrm{s}$. The dashed lines represent the first part of the orbit (before the impulse) and the continuous lines are the second part (after the impulse). Information about the trajectories: (a) $e=0.0$, $v=0^{\circ}, \psi=0^{\circ}$, and $\delta V=0.0 \mathrm{~km} / \mathrm{s} ;$ (b) $e=0.1, \alpha=1.0^{\circ}$ and $\Delta E_{\max }=20.625$ c.u.; (c) $e=0.3, \alpha=2.0^{\circ}$ and $\Delta E_{\max }=16.1766$ c.u.; (d) $e=0.5, \alpha=3.0^{\circ}$ and $\Delta E_{\max }=12.2308$ c.u. The symbol + represents the Moon.

\section{Acknowledgments}

The authors wish to express their appreciation for the support provided by Grants 304700/2009-6 from the National Council for Scientific and Technological Development (CNPq); Grants \#2011/13101-4, 2011/08171-3 and 2016/14665-2, from Fundação de Amparo à Pesquisa do Estado de São Paulo (FAPESP) and the financial support from the National Council for the Improvement of Higher Education (CAPES).

\section{References}

Armellin, R., Lavagna, M., Starkey, R.P., Lewis, M.J., 2007. Aerogravity assist maneuvers: coupled trajectory and vehicle shape optimization. J. Spacecraft Rock. 44, 1051-1059. http://dx.doi.org/10.2514/1.28713.

Bonfiglio, E.P., Longuski, J.M., Vinh, N.X., 2000. Automated design of aerogravity-assist trajectories. J. Spacecraft Rock. 37, 768-775. http:// dx.doi.org/10.2514/2.3649.

Brasil, P.I.O., Prado, A.F.B.A., Deienno, R., Yokoyama, T., 2015. Study of the gravitational capture of a spacecraft by Jupiter. Adv. Space Res. 55, 668-681. http://dx.doi.org/10.1016/j.asr.2014.11.005.
Broucke, R.A., 1969. Stability of periodic orbits in the elliptic, restricted three-body problem. AIAA J. 7, 1003-1009. http://dx.doi.org/10.2514/ 3.5267.

Broucke, R.A., 1988. The celestial mechanics of gravity assist. In: AIAA/ AAS Astrodynamics Conference, Minneapolis, MN, August 1988 (AIAA paper 88-4220), doi:http://dx.doi.org/10.2514/6.1988-4220.

Byrnes, D.V., D'Amario, L.A., 1982. A combined Halley flyby Galileo mission. In: AIAA/AAS Astrodynamics Conference, San Diego, CA, August 1982 (AIAA paper 82-1462), doi:http://dx.doi.org/10.2514/6. 1982-1462.

D'Amario, L.A., Byrnes, D.V., Stanford, R.H., 1981. A new method for optimizing multiple-flyby trajectories. J. Guid. Control Dynam. 4, 591-596. http://dx.doi.org/10.2514/3.56115.

D’Amario, L.A., Byrnes, D.V., Stanford, R.H., 1982. Interplanetary trajectory optimization with application to Galileo. J. Guid. Control Dynam. 5, 465-471. http://dx.doi.org/10.2514/3.56194.

Dowling, R.L., Kosmann, W.J., Minovitch, M.A., Ridenoure, R.W., 1991. Gravity propulsion research at UCLA and JPL 1962-1964. In: 41st Congress of the International Astronautical Federation, Dresden, GDR, 6-12 October 1991

Dowling, R.L., Kosmann, W.J., Minovitch, M.A., Ridenoure, 1992. R. W., Gravity propulsion research at UCLA and JPL 1962-1964. In: Congress of the International Astronautical Federation, 42, Montreal, Canada, October 1992. 
Ferreira, A.F.S., Prado, A.F.B.A., Winter, O.C., 2015. A numerical study of powered Swing-Bys around the Moon. Adv. Space Res. 56, 252272. http://dx.doi.org/10.1016/j.asr.2015.04.016.

Ferreira, A.F.S., Prado, A.F.B.A., Winter, O.C., 2016. Swing-By propulsado aplicado ao sistema de Haumea. In: Proceeding Series of the Brazilian Society of Computational and Applied Mathematics (DINCON 2015), vol. 4(1), doi:http://dx.doi.org/10.5540/03.2016. 004.01.0056.

Flandro, G., 1966. Fast reconnaissance missions to the outer solar system utilizing energy derived from the gravitational field of Jupiter. Acta Astronaut. 12 (4).

Formiga, J.K.S., Prado, A.F.B.A., 2014. Studying sequences of resonant orbits to perform successive close approaches with the Moon. J. Braz. Soc. Mech. Sci. Eng. 1, 1. http://dx.doi.org/10.1007/s40430-014-02548.

Gomes, V.M., Prado, A.F.B.A., 2010. A study of the impact of the initial energy in a close approach of a cloud of particles. WSEAS Trans. Math. 9, 811-820.

Gomes, V.M., Pineros, J.O.M., Prado, A.F.B.A., Golebiewska, J., 2015. Atmospheric close approaches with the Earth considering drag and lift forces. Comp. App. Mat. 1, 1-17. http://dx.doi.org/10.1007/s40314015-0256-x.

Grard, R., 2006. Mercury: the Messenger and BepiColombo missions a concerted approach to the exploration of the planet. Adv. Space Res. 38, 563. http://dx.doi.org/10.1016/j.asr.2006.06.015.

Jehn, R., Companys, V., Corral, C., Y'arnoz, D.G., Sánchez, N., 2008. Navigating BepiColombo during the weak-stability capture at Mercury. Adv. Space Res. 42, 1364-1369. http://dx.doi.org/10.1016/j. asr.2008.01.011.

Lavagna, M., Povoleri, A., Finzi, A.E., 2005. Interplanetary mission design with aero-assisted maneuvers multi-objective evolutive optimization. Acta Astronaut. 57, 498-509. http://dx.doi.org/10.1016/j. actaastro.2005.03.052.

Lewis, M.J., McRonald, A.D., 1992. Design of hypersonic waveriders for aero assisted interplanetary trajectories. J. Spacecraft Rock. 29, 653660. http://dx.doi.org/10.2514/3.11506.

Lohar, F.A., Misra, A.K., Mateescu, D., 1996. Optimal aero-gravity assist with heliocentric plane change. Acta Astronaut. 38, 445-456. http://dx. doi.org/10.1016/0094-5765(96)00017-3.

Lynam, A.E., Kloster, K.W., Longuski, J.M., 2011. Multiple-satelliteaided capture trajectories at Jupiter using the Laplace resonance. Celest. Mech. Dyn Astron. 109, 59-84.

McConaghy, T.T., Debban, T.J., Petropoulos, A.E., Longuski, J.M., 2003. Design and optimization of low-thrust gravity trajectories with gravity assist. J. Spacecraft Rock. 40, 380-387. http://dx.doi.org/ 10.2514/2.3973.

McNutt Jr., R.L., Solomon, S.C., Grard, R., Novara, M., Mukai, T., 2004. An international program for mercury exploration: synergy of
Messenger and Bepicolombo. Adv. Space Res. 33, 2126-2132. http:// dx.doi.org/10.1016/S0273-1177(03)00439-3.

McNutt Jr., R.L., Solomon, S.C., Gold, R.E., Leary, J.C., 2006. The messenger mission to mercury: development history and early mission status. Adv. Space Res. 38, 564-571. http://dx.doi.org/10.1016/j. asr.2005.05.044.

Minovitch, M.A., 1961. A method for determining interplanetary free-fall reconnaissance trajectories. JPL Tec. Memo 312-130, Pasadena.

NASA, 2010. Lcross - Lunar Crater Observation and Sensing Satellite LCROSS Overview. Page Editor: Robert Garner. Available from $<$ http://www.nasa.gov/missionpages/LCROSS/overview/index.html $>$.

NASA, 1999-2012. Messenger Trajectory, Developed by JHU/APL. Available from $<$ http://messenger.jhuapl.edu/themission $>$.

Nock, K.T., Upholf, C.W., 1979. Satellite aided orbit capture. AAS/ AIAA Paper, 79-165.

Okutsu, M., Yam, C.H., Longuski, J.M., 2006. Low-thrust trajectories to Jupiter via gravity assists from Venus, Earth and Mars. AIAA Paper 2006-6745. http://dx.doi.org/10.2514/6.2006-6745.

Prado, A.F.B.A., 1996. Powered Swing-By. J. Guid. Control Dynam. 19, 1142-1147. http://dx.doi.org/10.2514/3.21756.

Prado, A.F.B.A., 1997. Close-approach trajectories in the elliptic restricted problem. J. Guid. Control Dynam. 20, 797-802. http://dx. doi.org/10.2514/2.4115.

Ross, S.D., Scheeres, D.J., 2007. Multiple gravity assists, capture, and escape in the restricted three-body problem. J. Appl. Dynam. Syst. 6 (3), 576-596. http://dx.doi.org/10.1137/060663374.

Sanchez, D. M., Howell, K.C., Prado; A.F.B.A., 2016. On the dynamics of a spacecraft in the irregular Haumea-Hi'iaka binary. In: 26th Space Flight Mechanics Meeting, 2016. Napa.

Santos, D.P.S., Prado, A.F.B.A., Casalino, L., Colasurdo, G., 2008. Optimal trajectories towards near-earth-objects using Solar electric propulsion (sep) and gravity assisted maneuver. J. Aerosp. Eng., Sci. Appl. 1 (2), 51-64.

Silva, A.F., Prado, A.F.B.A., Winter, O.C., 2013a. Optimal impulsive control in a powered Swing-By. In: AIAA Guidance, Navigation, and Control (GNC) Conference, doi:http://dx.doi.org/10.2514/6.20134722.

Silva, A.F., Prado, A.F.B.A., Winter, O.C., 2013b. Powered Swing-By Maneuvers around the Moon. J. Phys. Conf. Ser. 465, 012001. http:// dx.doi.org/10.1088/1742-6596/465/1/012001.

Sims, J.A., Longuski, J.M., Patel, M.R., 1995. Aerogravity-assist trajectories to the outer planets. Acta Astronaut. 35, 297-306. http://dx.doi. org/10.1016/0094-5765(94)00195-R.

Sims, J.A., Longuski, J.M., Patel, M.R., 2000. Aerogravity-assist trajectories to the outer planets and the effect of drag. J. Spacecraft Rock. 37, 49-55. http://dx.doi.org/10.2514/2.3525.

Szebehely, V., 1967. Theory of Orbits. Academic Press, New York. 